\title{
Calculation of mutual information for nonlinear communication channel at large SNR
}

\author{
I. S. Terekhov, ${ }^{1,2, *}$ A. V. Reznichenko, ${ }^{1,2, \dagger}$ and S. K. Turitsyn ${ }^{3,2, \ddagger}$ \\ ${ }^{1}$ Budker Institute of Nuclear Physics of Siberian Branch Russian Academy of Sciences, Novosibirsk, 630090 Russia \\ ${ }^{2}$ Novosibirsk State University, Novosibirsk, 630090 Russia \\ ${ }^{3}$ Aston Institute of Photonics Technologies, Aston University, Aston Triangle, Birmingham, B4 $7 E T$, UK
}

(Dated: September 13, 2016)

\begin{abstract}
Using the path-integral technique we examine the mutual information for the communication channel modelled by the nonlinear Schrödinger equation with additive Gaussian noise. The nonlinear Schrödinger equation is one of the fundamental models in nonlinear physics, and it has a broad range of applications, including fiber optical communications - the backbone of the Internet. At large signal-to-noise ratio (SNR) we present the mutual information through the path-integral which is convenient for the perturbative expansion in nonlinearity. In the limit of small noise and small nonlinearity we derive analytically the first nonzero nonlinear correction to the mutual information for the channel.
\end{abstract}

PACS numbers: 05.10.Gg, 89.70.-a, 02.70.-c,02.70.Rr,05.90.+m

\section{INTRODUCTION}

There is a known link between entropy production in physical systems [1] and loss of information due to noise in communication channels [2]. Considering field (signal) evolution in dynamical system with noise, one can examine a continuous change of the mutual information between the initial and dynamically evolving fields (signals). The mutual information is a measure of the amount of information that can be obtained about one random variable (in this example - an initial field $X$ ) by observing another variable (here - the evolving field $Y$ ). The mutual information $I_{P[X]}$ (in continuousinput, continuous-output system) is expressed through the path-integral over input $X$ and output $Y$ fields:

$$
I_{P[X]}=\int \mathcal{D} X \mathcal{D} Y P[X] P[Y \mid X] \log \frac{P[Y \mid X]}{P_{\text {out }}[Y]},
$$

where $P[X]$ is the probability density function (PDF) of the initial signal $X$ with the fixed finite average power $P_{\text {ave }}$. The function $P[Y \mid X]$ in Eq. (1) is the conditional probability density function, that is the probability density of receiving output signal $Y$ when the input signal is $X$. The output signal PDF $P_{\text {out }}[Y]$ in Eq. (1) reads

$$
P_{\text {out }}[Y]=\int \mathcal{D} X P[X] P[Y \mid X] .
$$

Both signals $X$ and $Y$ may be discrete or continuous. When $X$ is discrete, the notation integral over $X$ stands for the summation of an under integral function over its discrete support. In the traditional communication systems functions $X$ and $Y$ usually have a bounded frequency supports, say, the signal $X(\omega)$ is not zero only

\footnotetext{
${ }^{*}$ E-mail: I.S.Terekhov@gmail.com

${ }^{\dagger}$ E-mail: A.V.Reznichenko@inp.nsk.su

${ }^{\ddagger}$ E-mail: s.k.turitsyn@aston.ac.uk
}

when $\omega \in W$ and $Y(\omega)$ is located within the interval $\omega \in \widetilde{W}$. In general, domains $W$ and $\widetilde{W}$ might be different, due to both nonlinear induced signal spreading in the channel, and filtering at the receiver (or inline).

Mutual information (1) is a difference between the entropy of the output signal

$$
H[Y]=-\int \mathcal{D} Y P_{\text {out }}[Y] \log P_{\text {out }}[Y]
$$

and the conditional entropy

$$
H[Y \mid X]=-\int \mathcal{D} X \mathcal{D} Y P[X] P[Y \mid X] \log P[Y \mid X] .
$$

When the signal and noise in the channel are independent variables and the received signal $Y$ is the sum of the transmitted signal $X$ and the noise, then it can be shown explicitly that the entropy of the output signal $H[Y]$ is greater than the entropy of the input signal

$$
H[X]=-\int \mathcal{D} X P[X] \log P[X]
$$

In this case, the transmission rate is the entropy of the received signal less the entropy $H[Y \mid X]$ which is due to impact of the noise. The maximal information transmission rate over a given bandwidth is given by the maximum of the functional $I_{P[X]}$ over input field distributions $P[X]$ and is referred to as the channel (Shannon) capacity $C$. This quantity was calculated for the linear channels with an additive white Gaussian noise (AWGN) in Ref. [2]:

$$
C \propto \log (1+\mathrm{SNR})
$$

where SNR is a signal-to-noise power ratio. This seminal theoretical result is the foundation of communication theory and it has proven its importance in a number of practical applications. To some extent, the Eq. (6) worked so well in so many situations that some engineers cease to 
distinguish the general Shannon expression for capacity and particular result for the specific linear channel with AWGN (6).

Recent advances in optical fiber communications where the channel is nonlinear, as opposite to the linear channel with AWGN, attracted interest to calculation of the Shannon capacity for nonlinear channels. To increase the channel capacity over a certain bandwidth with a given accumulated noise of optical amplifiers, one has to increase the signal power, see (6). This works in the low SNR limit but the refraction index dependence of the fibers on light intensity (the Kerr effect) dramatically changes the propagation properties of the fiber optical channel at higher signal power. In other words, the optical fiber channel becomes nonlinear at high light intensity.

Recent studies have shown that the spectral efficiency (that is, the number of bits, or nats, transmitted per second per Hertz - practical characteristics having the same dimension as the channel capacity per spectral unit) of a fiber optical channel is limited by the Kerr nonlinearity. These studies indicated that observable spectral efficiency always turns out to be less than the Shannon limit of the corresponding linear channel with AWGN (6) [37]. It has been observed that the spectral efficiency of the nonlinear channel decreases with increasing SNR at high enough values of SNR [3-5, 7]. This analysis certainly provides only a lower bound on channel capacity and does not prove that the Shannon nonlinear fiber channel capacity is decreasing with power; see, for example, discussions in [8-12]. As a matter of fact, the decrease of the spectral efficiency can be linked to different effects. The first effect is the nonlinear interaction of the signal with noise, which leads to effective increase of the noise power. The second one is the leak of the signal power out of the filter domain $\widetilde{W}$ even for zero noise case, i.e. not complete collection of the transmitted signal at the receiver.

In [8] it was shown that the capacity of certain nonlinear channels could not decrease with SNR. Also for the nondispersive nonlinear channel it was shown that the channel capacity is growing with increasing SNR, see Refs. [11-13]. However, the capacity of nonlinear fiber channels is still an open problem of great practical and fundamental importance. Therefore, it is important to develop new techniques and mathematical methods to study this problem, especially in the most important case of large SNR.

The fiber optical channel contains the fiber links where the signal propagates and the signal amplitude decreases, and amplifiers which compensate the amplitude decreasing. Also the amplifiers are the noise source in the channel. In the series of papers [14-17] it was shown that such fiber optic channel can be described by the nonlinear Schrödinger equation (NLSE) with additive white Gaussian noise. In the present paper we calculate analytically the mutual information for the channel described by the nonlinear Schrödinger equation with AWGN in the leading nonzero order in nonlinearity and at large SNR. We demonstrate that the first nonlinear correction for the channel with dispersion is negative and it is quadratic in the Kerr nonlinearity parameter. We compare our result for the mutual information in the case of the channel with nonzero dispersion and the exact result for the nonlinear nondispersive channel. We show that there is the region of the parameter SNR where the obtained mutual information is greater than that obtained for the channel with zero dispersion. We also show that the region becomes wider with increasing of the dispersion parameter.

The article is organized as follows: in the Section II we consider the channel model and the general structure of the conditional probability density function. In the Section III we obtain the general expressions for the output signal entropy, conditional entropy and the mutual information. The Section IV is focused on the calculation of the first nonlinear correction to the mutual information and comparison of the result obtained with that for the nondispersive channel. In the Conclusion we discuss our results. The details of calculation are presented in Appendixes.

\section{NONLINEAR CHANNEL MODEL AND THE CONDITIONAL PROBABILITY AT SMALL NOISE POWER}

In our model the propagation of the signal $\psi(z, t)$ is described by the NLSE with AWGN, see [14-17]:

$$
\partial_{z} \psi+i \beta \partial_{t}^{2} \psi-i \gamma|\psi|^{2} \psi=\eta(z, t),
$$

where $\beta$ is the dispersion coefficient, $\gamma$ is the Kerr nonlinearity coefficient, $\eta(z, t)$ is an additive complex white noise with zero mean $\langle\eta(z, t)\rangle_{\eta}=0$ and correlation function

$$
\left\langle\eta(z, t) \bar{\eta}\left(z^{\prime}, t^{\prime}\right)\right\rangle_{\eta}=Q \delta\left(z-z^{\prime}\right) \delta\left(t-t^{\prime}\right),
$$

where bar means complex conjugation, and $Q$ is a power of the white Gaussian noise $\eta(z, t)$ per unit length and per unit frequency. The initial condition for the signal $\psi(z, t)$ is $\psi(z=0, t)=X(t)$ and we define: $\psi(z=L, t)=Y(t)$. Here $L$ is signal propagation distance. As we mentioned previously we consider the case where the input signal $X$ has the bounded frequency support $W$. Therefore it is convenient to consider the problem in the frequency domain. Any functions in the time and frequency domains are related as follows: $f(z, t)=\int \frac{d \omega}{2 \pi} e^{-i \omega t} f_{\omega}(z)$. In the frequency domain our Eqs. (7) and (8) have the form:

$$
\begin{aligned}
& \partial_{z} \psi_{\omega}(z)-i \beta \omega^{2} \psi_{\omega}(z)- \\
& i \gamma \int_{-\infty}^{\infty} \frac{d \omega_{1} d \omega_{2}}{(2 \pi)^{2}} \psi_{\omega_{1}}(z) \psi_{\omega_{2}}(z) \bar{\psi}_{\omega_{3}}(z)=\eta_{\omega}(z),
\end{aligned}
$$

where $\omega_{3}=\omega_{1}+\omega_{2}-\omega$,

$$
\left\langle\eta_{\omega}(z) \bar{\eta}_{\omega^{\prime}}\left(z^{\prime}\right)\right\rangle_{\eta}=2 \pi Q \delta\left(z-z^{\prime}\right) \delta\left(\omega-\omega^{\prime}\right) \chi_{W^{\prime}}(\omega),
$$


where $\chi_{W^{\prime}}(\omega)=\theta\left(W^{\prime} / 2-\omega\right) \theta\left(W^{\prime} / 2+\omega\right)$, with $\theta(x)$ being Heaviside $\theta$-function. Strictly speaking the finite frequency domain of the noise means that the noise is not white having the finite frequency support $W^{\prime}$. But if $W^{\prime}$ is much larger than the frequency domain of the signal $\psi_{\omega}(z)$ (i.e. $W^{\prime} \gg W$ and $W^{\prime} \gg \widetilde{W}$ ) then the noise can be treated as a white one. Our results will not depend on the parameter $W^{\prime}$ and at the final stage we consider infinitely large $W^{\prime}$ (true white noise). It is worth emphasizing that in a nonlinear channel transmitted and received signal bandwidths can differ from each other. Therefore, we assume here that in general, the input $X(\omega)$ and output $Y(\omega)$ signals have frequency domains $[-W / 2, W / 2]$ and $[-\widetilde{W} / 2, \widetilde{W} / 2]$ respectively.

The model of the input signal $X$. We imply that the input signal $X(\omega)$ is not zero in the frequency domain $W$ and $X(\omega)=0$ in the domain $W^{\prime} \backslash W$. In the domain $W$ the signal $X(\omega)$ has the PDF with zero mean and with fixed average power. Since $X(\omega)$ in the domain $W^{\prime} \backslash W$ is defined and is equal to zero the PDF has the form of delta-function in the domain. Therefore one has

$$
P[X(\omega)]=P_{X}^{(M)}[X(\omega)] \prod_{j \in W^{\prime} \backslash W}^{M^{\prime}-M} \delta\left(X_{j}\right) .
$$

Here we divide the domain $W^{\prime}$ into $M^{\prime}$ equal intervals and the domain $W$ into $M$ equal intervals. The form (11) stands for the fact that we have $M$ independent complex meaning channels in the domain $W$ with the same PDF in every channel:

$$
P_{X}^{(M)}[X(\omega)]=\prod_{j=1}^{M} P\left[X_{j}\right] .
$$

Here $\delta\left(X_{j}\right)=\delta\left(\operatorname{Re} X_{j}\right) \delta\left(\operatorname{Im} X_{j}\right)$ is the $\delta$-function, $X_{j}=$ $X\left(\omega_{j}\right)$. The frequency domain $W\left(W^{\prime}\right)$ is divided by $M$ $\left(M^{\prime}\right)$ grids spacing $\delta=W /(2 \pi M)=W^{\prime} /\left(2 \pi M^{\prime}\right)$. The distribution (11) means that there are $M$ elementary independent complex coefficients presenting information in the spectral domain $W$. The average power for $P[X(\omega)]$ reads

$$
P_{\text {ave }}=\lim _{T \rightarrow \infty} \int \mathcal{D} X P[X(\omega)] \int_{W^{\prime}} \frac{d \omega}{2 \pi T}|X(\omega)|^{2}=\frac{P W}{2 \pi},
$$

where $T$ is the time interval containing the whole input signal in the time domain. We will use the relation $M=T W / 2 \pi$ that corresponds to the Nyquist-ShannonKotelnikov theorem [18]. In Eq. (13) we have introduced quantity $P$ that is the power per unit frequency (spectral power density), it means that the average power in one elementary spectral step is $P \delta$. The measure in Eq. (13) $\mathcal{D} X=\prod_{j=1}^{M^{\prime}} d \operatorname{Re} X_{j} d \operatorname{Im} X_{j}$ is consistent with the normalization condition

$$
\int \mathcal{D} X P[X(\omega)]=1
$$

For typical fiber optical links the ratio $\mathrm{SNR}=P /(Q L)$ is of order of $10^{4}$. Therefore, in what follows we assume that the parameter $P$ is much greater than the accumulated noise power $Q L$ in the channel (large SNR case):

$$
P \gg Q L
$$

In Ref. [19] using the methods described in Refs. [2022 ] the conditional probability density $P[Y(\omega) \mid X(\omega)]$ for our channel model was found. It was shown that the quantity $P[Y(\omega) \mid X(\omega)]$ can be expressed through the path-integral:

$$
\begin{gathered}
P[Y(\omega) \mid X(\omega)]=\Lambda e^{-S\left[\Psi_{\omega}(z)\right] / Q}, \\
\Lambda=\int_{\phi_{\omega}(0)=0}^{\phi_{\omega}(L)=0} \mathcal{D} \phi e^{-\left\{S\left[\Psi_{\omega}(z)+\phi_{\omega}(z)\right]-S\left[\Psi_{\omega}(z)\right]\right\} / Q} .
\end{gathered}
$$

Here the functional $S[\psi]$ is referred to as the action, and it has the form

$$
\begin{aligned}
& S[\psi]=\int_{0}^{L} d z \int_{W^{\prime}} \frac{d \omega}{2 \pi} \mid \partial_{z} \psi_{\omega}(z)-i \beta \omega^{2} \psi_{\omega}(z)- \\
& \left.i \gamma \int_{W^{\prime}} \frac{d \omega_{1} d \omega_{2}}{(2 \pi)^{2}} \psi_{\omega_{1}}(z) \psi_{\omega_{2}}(z) \bar{\psi}_{\omega_{3}}(z)\right|^{2},
\end{aligned}
$$

where $\omega_{3}=\omega_{1}+\omega_{2}-\omega$. The function $\Psi_{\omega}(z)$ in Eq. (16) is referred to as the "classical trajectory". It is the extremum function of the action $S$, i.e. the action variation is equal to zero on the function $\Psi_{\omega}(z): \delta S[\Psi]=0$ with the boundary conditions $\Psi_{\omega}(0)=X(\omega), \Psi_{\omega}(L)=Y(\omega)$. We omit here the explicit form of the equation because it is quite cumbersome, but one can find it in the Appendix A, see Eq. (A10). The path-integral in Eq. (17) is defined in the discretization scheme that takes into account the casuality principle, see details in Ref. [19]. The measure $\mathcal{D} \phi$ in Eq. (17) is defined as

$$
\mathcal{D} \phi=\lim _{\delta \rightarrow 0} \lim _{\Delta \rightarrow 0}\left(\frac{\delta}{\Delta \pi Q}\right)^{N M^{\prime}} \prod_{j=1}^{M^{\prime}} \prod_{i=1}^{N-1} d \phi_{i, j},
$$

where $d \phi_{i, j}=d \operatorname{Re} \phi_{i, j} d \operatorname{Im} \phi_{i, j}, \phi_{i, j}=\phi_{\omega_{j}}\left(z_{i}\right), \Delta=$ $L / N$ is the coordinate grids spacing, $\delta$ is the frequency grids spacing introduced after Eq. (12). The measure (19) is consistent with the normalization condition

$$
\int \mathcal{D} Y P[Y(\omega) \mid X(\omega)]=1,
$$

where the measure $\mathcal{D} Y$ is defined as

$$
\mathcal{D} Y=\prod_{j=1}^{M^{\prime}} d \operatorname{Re} Y_{j} d \operatorname{Im} Y_{j}, \quad Y_{j}=Y\left(\omega_{j}\right) .
$$

Let us now consider the function $P[Y(\omega) \mid X(\omega)]$ at small $Q$. Our consideration of the $P[Y \mid X]$ at small parameter $Q$ is similar to the quasi-classical approximation 
in the quantum mechanics at small Planck's constant $\hbar$ [23]. Let us consider what output signals $Y(\omega)$ are statistically significant for $P[Y(\omega) \mid X(\omega)]$ at given $X(\omega)$, i.e. when $S[\Psi]$ is less or of order of $Q$. The physical picture is as follows. At small $Q$ the trajectory $\Psi_{\omega}(z)$ can not be sufficiently different from $\Phi_{\omega}(z)$ which is the solution of Eq. (9) with zero noise $\eta=0$ and with the boundary condition $\Phi_{\omega}(0)=X(\omega)$. When solving (9) with the fixed $X(\omega)$ and nonzero (but small) noise $\eta$ we can expect that the solution at $z=L, \psi_{\omega}(L)$, has the difference from $\Phi_{\omega}(z=L)$ that is proportional to $\sqrt{Q}$ because the average noise power per unit frequency is small, see Eqs. (10), (15). That is why the difference $Y(\omega)-\Phi_{\omega}(L)$ should be proportional to $\sqrt{Q L}$. Thus, for such $Y(\omega)$ we can seek the solution $\Psi_{\omega}(z)$ as the series in parameter $\sqrt{Q L}$ :

$$
\Psi_{\omega}(z)=\Phi_{\omega}(z)+\varkappa_{\omega}(z), \quad \varkappa_{\omega}(z)=\sum_{n=1}^{\infty} \varkappa_{\omega}^{(n)}(z)
$$

where $\varkappa_{\omega}^{(n)}(z) \propto(Q L)^{n / 2}$. Inserting the solution (22) in the action $S[\Psi]$ and taking into account that $S[\Phi]=0$ we obtain $S[\Psi]=S_{2}\left[\varkappa^{(1)}\right]+\widetilde{S}[\varkappa]$, where $S_{2}\left[\varkappa^{(1)}\right]$ is the quadratic functional in $\varkappa^{(1)}$, i.e. $S_{2}\left[\varkappa^{(1)}\right] \propto Q$, and $\widetilde{S}[\varkappa]$ is the reminder functional that is suppressed in the parameter $Q$ (its expansion in $Q$ starts from $Q^{3 / 2}$ ). In what follows we are interested only in the leading order in parameter $Q L$, therefore:

$$
\begin{aligned}
& S[\Psi] \approx S_{2}\left[\varkappa^{(1)}\right]=\int_{0}^{L} d z \int_{W^{\prime}} \frac{d \omega}{2 \pi} \mid \partial_{z} \varkappa_{\omega}^{(1)}-i \beta \omega^{2} \varkappa_{\omega}^{(1)}- \\
& \left.i \gamma \int_{W^{\prime}} \frac{d \omega_{1} d \omega_{2}}{(2 \pi)^{2}}\left(2 \varkappa_{\omega_{1}}^{(1)} \Phi_{\omega_{2}} \bar{\Phi}_{\omega_{3}}+\bar{\varkappa}_{\omega_{3}}^{(1)} \Phi_{\omega_{1}} \Phi_{\omega_{2}}\right)\right|^{2}
\end{aligned}
$$

where $\omega_{3}=\omega_{1}+\omega_{2}-\omega$. The function $\varkappa_{\omega}^{(1)}(z)$ obeys the linear equation with coefficients depending on $\Phi_{\omega}(z)$ with the boundary conditions $\varkappa_{\omega}^{(1)}(0)=0, \varkappa_{\omega}^{(1)}(L)=$ $Y(\omega)-\Phi_{\omega}(L)$. The equation for $\varkappa^{(1)}$ has a compact form in the time domain:

$$
\begin{aligned}
& \left(\partial_{z}+i \beta \partial_{t}^{2}-2 i \gamma|\Phi|^{2}\right) l\left[\varkappa^{(1)}\right]+i \gamma \Phi^{2} \bar{l}\left[\varkappa^{(1)}\right]=0 \\
& l[\varkappa]=\left(\partial_{z}+i \beta \partial_{t}^{2}\right) \varkappa-i \gamma\left(2|\Phi|^{2} \varkappa+\Phi^{2} \bar{\varkappa}\right)
\end{aligned}
$$

This equation in the frequency domain is cumbersome, therefore, we do not present it here but one can find it in the Appendix A, see Eq. (A12). Since the Eq. (24) is linear in $\varkappa^{(1)}$ the solution of the equation for $\varkappa_{\omega}^{(1)}(z)$ linearly depends on its value $\delta Y(\omega)=Y(\omega)-\Phi_{\omega}(L)$ on the boundary $z=L$. Since the action (23) is quadratic functional in $\varkappa_{\omega}^{(1)}(z)$ we can write

$$
S[\Psi] \approx \int d \omega d \omega^{\prime} \delta Y^{(\alpha)}(\omega) \mathcal{L}_{\alpha, \beta}\left(\omega, \omega^{\prime}\right) \delta Y^{(\beta)}\left(\omega^{\prime}\right),
$$

where $\delta Y^{(1)}(\omega)=\operatorname{Re} \delta Y(\omega), \delta Y^{(2)}(\omega)=\operatorname{Im} \delta Y(\omega)$, and $\mathcal{L}_{\alpha, \beta}\left(\omega, \omega^{\prime}\right),(\alpha, \beta=1,2)$ is some integral kernel that depends on function $\Phi_{\omega}(z)$. Note that the solution $\Phi_{\omega}(z)$ of Eq. (9) can be written as $\Phi_{\omega}(L)=(\hat{L} X)(L, \omega) \equiv \hat{L} X$, where $\hat{L}$ is the nonlinear evolution operator of Eq. (9), see [24]. It means that in the leading order in $Q$ the kernel $\mathcal{L}_{\alpha, \beta}\left(\omega, \omega^{\prime}\right)$ depends on input signal $X(\omega)$ rather than $Y(\omega)$. The representation (26) is valid for arbitrary nonlinearity but in the leading order in $Q$.

Let us consider the normalization factor $\Lambda$ in Eq. (17). In order to calculate $\Lambda$ in the leading order in $Q$ we should keep only the quadratic in $\phi_{\omega}(z)$ terms in the action difference, see Eq. (17). Using Laplace's method applied to the path-integral one can show that the higher powers of $\phi_{\omega}(z)$ result in the suppressed corrections in the parameter $Q$. The coefficients in the quadratic in $\phi_{\omega}(z)$ terms in the action difference depend on the function $\Psi$, but in the leading order in $Q$ we can substitute $\Phi$ instead of $\Psi$. It means that in this order the normalization factor $\Lambda=\Lambda[X]$ depends only on $X(\omega)$. In the leading order in $Q$ the factor $\Lambda[X]$ can be found in several ways: by the direct calculation of the path-integral or by using the normalization condition (20). The latter reads

$$
\int \mathcal{D} Y P[Y \mid X]=\Lambda[X] \int \mathcal{D} \delta Y e^{-S\left[\Psi_{\omega}(z)\right] / Q}=1
$$

In the discrete form the functions $X(\omega)$ and $Y(\omega)$ can be presented as $2 M^{\prime}$-dimensional real vectors $\vec{X}$ and $\vec{Y}$, respectively, which describe both real and imaginary part of these quantities on the frequency grid. Thus Eq. (26) reads in the discretization as follows:

$$
S[\Psi] \approx \delta^{2} \delta \vec{Y}^{\dagger} \mathcal{L} \delta \vec{Y}
$$

with $\mathcal{L}=\mathcal{L}[\vec{X}]$ being $2 M^{\prime} \times 2 M^{\prime}$-dimensional Hermitian matrix depending on $\vec{X}$ only. For $\Lambda[X]$ one has

$$
\Lambda[X]=\sqrt{\operatorname{det}[\mathcal{L}]}\left(\delta^{2} /(\pi Q)\right)^{M^{\prime}} .
$$

Therefore in the leading order in $Q$ the conditional probability density function $P[Y \mid X]$ has the form:

$$
P[Y \mid X]=\Lambda[X] e^{-\delta^{2} \delta \vec{Y}^{\dagger} \mathcal{L} \delta \vec{Y} / Q} .
$$

The conditional probability density function $P[Y \mid X]$ must obey the restriction $[12,19]$ :

$$
\lim _{Q \rightarrow 0} P[Y \mid X]=\delta(\vec{Y}-\overrightarrow{\hat{L} X})
$$

that is nothing more but the deterministic limit of zero noise. In our approximation for the $P[Y \mid X]$ this condition (31) is fulfilled automatically due to the exponential form (30) and normalization factor (29). Now we can move to the consideration of the output and conditional signal entropies (3) and (4), respectively. 


\section{ENTROPIES AND MUTUAL INFORMATION}

First we consider the PDF $P_{\text {out }}[Y]$, see Eq. (2). To begin with we perform the decomposition of any $2 M^{\prime}$ vector $(\vec{X}, \vec{Y}$, etc. $) \quad \vec{V}=\vec{V}_{1} \oplus \vec{V}_{2}$, where $\vec{V}_{1}$ is $2 M$ dimensional vector corresponding to $M$ meaning complex channels in the frequency domain $W$, whereas $\vec{V}_{2}$ is $2\left(M^{\prime}-M\right)$-dimensional vector corresponding to remnant $M^{\prime}-M$ complex channels in the frequency domain $W^{\prime} \backslash W$. The sign $\oplus$ means the direct sum. We substitute the PDF $P[X(\omega)]$ in the form (11) and the conditional PDF $P[Y \mid X]$ in the form (30) into the definition (2) and obtain $P_{\text {out }}[\vec{Y}]$ in the discretization scheme:

$$
\begin{aligned}
P_{\text {out }}[\vec{Y}]= & \int d \vec{X}_{1} d \vec{X}_{2} P_{X}^{(M)}\left[\vec{X}_{1}\right] \delta\left(\vec{X}_{2}\right) \times \\
& \Lambda[\vec{X}] e^{-\delta^{2} \delta \vec{Y}^{\dagger} \mathcal{L} \delta \vec{Y} / Q},
\end{aligned}
$$

where $\delta \vec{Y}=\vec{Y}-\overrightarrow{\hat{L} X}, \delta\left(\vec{X}_{2}\right)$ means $2\left(M^{\prime}-M\right)$ dimensional delta-function. For the following calculation it is convenient to perform the transformation of the action (28). We can write $\vec{X}=\overrightarrow{\hat{L}^{-1} Y}-J \delta \vec{Y}+\mathcal{O}\left(\delta \vec{Y}^{2}\right)$ using that $\delta \vec{Y} \sim \sqrt{Q}$. Here $J_{i, i^{\prime}}=\partial \hat{L}^{-1} Y_{i} / \partial Y_{i^{\prime}}$ is the Jacobian matrix of the mapping $\hat{L}^{-1}, i, i^{\prime}=1, \ldots, 2 M^{\prime}$. Since the Jacobian $\operatorname{det}[J]$ has the unit absolute value, see [24], we can write $\delta \vec{Y}=-J^{-1} \vec{Z}$, where $\vec{Z}=\vec{X}-\overrightarrow{\hat{L}^{-1} Y}$. Now we change variables in Eq. (32) from $\vec{X}$ to $\vec{Z}=\vec{Z}_{1} \oplus \vec{Z}_{2}$. In the new variables the action (28) reads $S[\Psi] \approx \delta^{2} \vec{Z}^{\dagger} \mathcal{K} \vec{Z}$, where Hermitian matrix $\mathcal{K}=J^{-1 \dagger} \mathcal{L} J^{-1}$ has the block form

$$
\mathcal{K}=J^{-1 \dagger} \mathcal{L} J^{-1}=\left(\begin{array}{ll}
\mathcal{K}_{11}, & \mathcal{K}_{12} \\
\mathcal{K}_{21}, & \mathcal{K}_{22}
\end{array}\right) .
$$

Here the block $\mathcal{K}_{11}$ is $2 M \times 2 M$ matrix, $\mathcal{K}_{12}$ is $2 M \times$ $2\left(M^{\prime}-M\right)$ matrix, $\mathcal{K}_{12}=\mathcal{K}_{21}^{\dagger}$, the block $\mathcal{K}_{22}$ is $2\left(M^{\prime}-\right.$ $M) \times 2\left(M^{\prime}-M\right)$ matrix. In the new variables the action (28) has the form

$$
\begin{aligned}
S[\Psi] \approx & \left(\vec{Z}_{1}+\mathcal{K}_{11}^{-1} \mathcal{K}_{12} \vec{Z}_{2}\right)^{\dagger} \mathcal{K}_{11}\left(\vec{Z}_{1}+\mathcal{K}_{11}^{-1} \mathcal{K}_{12} \vec{Z}_{2}\right) \delta^{2}+ \\
& \vec{Z}_{2}^{\dagger}\left(\mathcal{K}_{22}-\mathcal{K}_{21} \mathcal{K}_{11}^{-1} \mathcal{K}_{12}\right) \vec{Z}_{2} \delta^{2}
\end{aligned}
$$

where matrices $\mathcal{K}_{\alpha \beta}$ depend on $\vec{X}=\vec{Z}+\overrightarrow{\hat{L}^{-1} Y}$. Now we can perform the integration over $d \vec{Z}_{2}$, everywhere substituting $-\overrightarrow{\hat{L}}^{-1} Y_{2}$ instead of $\vec{Z}_{2}$ in view of the delta-function. Passing to new variables $\vec{Z}_{1}^{\prime}=\vec{Z}_{1}-$ $\mathcal{K}_{11}^{-1} \mathcal{K}_{12}{\overrightarrow{\hat{L}^{-1} Y}}_{2}$ we obtain in the leading order in $1 / \mathrm{SNR}$

$$
\begin{aligned}
& P_{\text {out }}[\vec{Y}]=\int d \vec{Z}_{1}^{\prime} P_{X}^{(M)}\left[\vec{Z}_{1}^{\prime}+{\overrightarrow{\hat{L}^{-1} Y_{1}}}+\mathcal{K}_{11}^{-1} \mathcal{K}_{12} \overrightarrow{\hat{L}}^{-1} Y_{2}\right] \times \\
& \Lambda\left[\vec{Z}_{1}^{\prime}+{\overrightarrow{\hat{L}^{-1} Y_{1}}}+\mathcal{K}_{11}^{-1} \mathcal{K}_{12}{\overrightarrow{\hat{L}^{-1} Y_{2}}}_{2}\right] e^{-\delta^{2} \vec{Z}_{1}^{\prime \dagger} \mathcal{K}_{11} \vec{Z}_{1}^{\prime} / Q} \times \\
& \exp \left[-\frac{\delta^{2}}{Q} \overrightarrow{\hat{L}^{-1} Y_{2}^{\dagger}}\left(\mathcal{K}_{22}-\mathcal{K}_{21} \mathcal{K}_{11}^{-1} \mathcal{K}_{12}\right){\overrightarrow{\hat{L}^{-1} Y_{2}}}_{\text {. }}\right.
\end{aligned}
$$

Since the first exponent $e^{-\delta^{2} \vec{Z}_{1}^{\prime \dagger} \mathcal{K}_{11} \vec{Z}_{1}^{\prime} / Q}$ in Eq. (35) is essentially narrower than the function $P_{X}^{(M)}[X]$, see Eq. (15), we can set $\vec{Z}_{1}^{\prime}=0$ in the argument of $P_{X}^{(M)}[X]$ and in the argument of $\Lambda[X]$. The second exponent in Eq. (35) demonstrates that $\overrightarrow{\hat{L}}^{-1} Y_{2} \propto \sqrt{Q}$ as well, therefore in the leading order in $1 / \mathrm{SNR}$ we can omit $\overrightarrow{\hat{L}}^{-1} Y_{2}$ in the arguments of $P_{X}^{(M)}[X]$ and $\Lambda[X]$. After this simplifications we perform the Gaussian integration over $\vec{Z}_{1}^{\prime}$ and finally obtain

$$
\begin{aligned}
& P_{\text {out }}[\vec{Y}]=P_{X}^{(M)}\left[\overrightarrow{\hat{L}}^{-1} Y_{1}\right] \times \\
& \Lambda_{2} e^{-\delta^{2} \overrightarrow{\hat{L}}^{-1} Y_{2}^{\dagger}}\left(\mathcal{K}_{22}-\mathcal{K}_{21} \mathcal{K}_{11}^{-1} \mathcal{K}_{12}\right){\overrightarrow{\hat{L}}-1 Y_{2} / Q}^{P^{-1}}
\end{aligned}
$$

where

$$
\Lambda_{2}=\sqrt{\operatorname{det}\left[\mathcal{K}_{22}-\mathcal{K}_{21} \mathcal{K}_{11}^{-1} \mathcal{K}_{12}\right]}\left(\delta^{2} /(\pi Q)\right)^{M^{\prime}-M} .
$$

Here the matrices $\mathcal{K}_{\alpha \beta}$ depend on vector $\overrightarrow{\hat{L}}^{-1} Y_{1}$. To obtain Eq. (36) we have used the factorization identity

$$
\Lambda[X]=\Lambda_{1}[X] \times \Lambda_{2}[X]
$$

where $\Lambda[X]$ is given by Eq. (29), i.e. $\Lambda[X]=$ $\sqrt{\operatorname{det}[\mathcal{L}]}\left(\delta^{2} /(\pi Q)\right)^{M^{\prime}}=\sqrt{\operatorname{det}[\mathcal{K}]}\left(\delta^{2} /(\pi Q)\right)^{M^{\prime}}$, and

$$
\Lambda_{1}[X]=\sqrt{\operatorname{det}\left[\mathcal{K}_{11}\right]}\left(\delta^{2} /(\pi Q)\right)^{M} .
$$

Let us note that $P_{\text {out }}[\vec{Y}]$ obtained in the leading order in $1 / \mathrm{SNR}$, see Eq. (36), is a product of the initial signal PDF $P_{X}^{(M)}$ in $M$ complex meaning channels and some noise distribution in the other $M^{\prime}-M$ complex channels which depends on the signal $\overrightarrow{\hat{L}}^{-1} Y_{1}$ of the meaning channels through the matrices $\mathcal{K}_{\alpha \beta}$.

Now we can calculate the output signal entropy $H[Y]$, see Eq. (3). To this end we insert $P_{\text {out }}$ in the form (36) to the Eq. (3), then change the integration variables from $\vec{Y}$ to $\vec{N}=\overrightarrow{\hat{L}^{-1} Y}$. Next using the fact that the Jacobian $\operatorname{det}[J]$ has the unit absolute value we perform integration over $\vec{N}_{2}$ and obtain:

$$
\begin{aligned}
& H[Y]=H[X]+\left(M^{\prime}-M\right)- \\
& \int d \vec{N}_{1} P_{X}^{(M)}\left[\vec{N}_{1}\right] \log \Lambda_{2}\left[\vec{N}_{1}\right]
\end{aligned}
$$

where

$$
H[X]=-\int d \vec{N}_{1} P_{X}^{(M)}\left[\vec{N}_{1}\right] \log P_{X}^{(M)}\left[\vec{N}_{1}\right]
$$

is the entropy of the input signal $X$, see Eq. (5).

Next, we calculate the conditional entropy $H[Y \mid X]$, see Eq. (4). This calculation is similar to one performed above. First, we perform the integration over $\vec{X}_{2}$. Then we change the variables $\vec{X}_{1}$ to $\vec{Z}_{1}^{\prime}=\vec{X}_{1}-$ 
$\overrightarrow{\hat{L}}^{-1} Y_{1}-\mathcal{K}_{11}^{-1} \mathcal{K}_{12} \overrightarrow{\hat{L}}^{-1} Y_{2}$. Then we change the variables $\vec{Y}$ to $\vec{N}=\overrightarrow{\hat{L}^{-1} Y}$. After that we perform integration over $\vec{N}$ and then over $\vec{Z}_{1}^{\prime}$. Finally, we obtain the conditional entropy $H[Y \mid X]$ in the leading order in 1/SNR:

$$
\begin{aligned}
H[Y \mid X]= & M^{\prime}-\int d \vec{Z}_{1}^{\prime} P_{X}^{(M)}\left[\vec{Z}_{1}^{\prime}\right] \log \Lambda_{1}\left[\vec{Z}_{1}^{\prime}\right]- \\
& \int d \vec{Z}_{1}^{\prime} P_{X}^{(M)}\left[\vec{Z}_{1}^{\prime}\right] \log \Lambda_{2}\left[\vec{Z}_{1}^{\prime}\right] .
\end{aligned}
$$

To obtain the mutual information (1) we subtract $H[Y \mid X]$, see Eq. (42), from $H[Y]$, see Eq. (40), and get

$$
I_{P[X]}=H[X]-M+\int d \vec{Z}_{1}^{\prime} P_{X}^{(M)}\left[\vec{Z}_{1}^{\prime}\right] \log \Lambda_{1}\left[\vec{Z}_{1}^{\prime}\right]
$$

Note that the mutual information $I_{P[X]}$ depends only on $M$ complex coefficients (meaning channels), whereas the entropies (40) and (42) depend on $M^{\prime}$ complex parameters. One can see that in the leading order in $1 / \mathrm{SNR}$ our result (43) contains the initial signal entropy $H[X]$ and the logarithm of the normalization factor $\Lambda_{1}$ averaged over the initial signal distribution $P_{X}^{(M)}$. Therefore to calculate $I_{P[X]}$ we have to know the normalization factor $\Lambda_{1}$.

\section{FIRST NONLINEAR CORRECTION}

In this section we consider the mutual information (43) in different regimes in the case when the input signal PDF $P_{X}^{(M)}$ has the Gaussian form. First, we examine the mutual information in the limit of small nonlinearity: when the dimensionless parameter $\tilde{\gamma}=P_{\text {ave }} \gamma L$ is small. In this case we calculate the first nonzero nonlinear correction to the mutual information for the arbitrary dispersion parameter $\beta$. Secondly, we consider the mutual information for the arbitrary nonlinearity and zero dispersion.

To find the mutual information (43) at small $\tilde{\gamma}$ we should calculate the initial signal entropy $H[X]$, see Eq. (41), and the normalization factor $\Lambda_{1}$. We use the Gaussian input signal PDF $P_{X}^{(M)}$ in the form

$$
P_{X}^{(M)}\left[\vec{X}_{1}\right]=P_{G}\left[\vec{X}_{1}\right]=\Lambda_{P} e^{-\left|\vec{X}_{1}\right|^{2} \delta / P},
$$

where $\Lambda_{P}$ is consistent with the normalization condition (14) and has the form:

$$
\Lambda_{P}=(\delta /(\pi P))^{M} .
$$

The input signal PDF in the form (44) means that the average signal power (13) is $P_{\text {ave }}=P W /(2 \pi) \gg$ $P_{\text {noise }}=Q L W /(2 \pi)$. The normalization condition reads $\int d \vec{X}_{1} P_{G}\left[\vec{X}_{1}\right]=1$. Substitution of the PDF (44) into Eq. (41) and the following integration yields:

$$
H[X]=M+M \log (\pi P / \delta) .
$$

To calculate the averaged $\log \Lambda_{1}$ over PDF $P_{G}\left[\vec{X}_{1}\right]$ in Eq. (43) in the leading and next-to-leading order in $\tilde{\gamma}$ we have to factorize $\Lambda$ in the form of the path-integral Eq. (17), see Eq. (38). We divide the integration region $W^{\prime}$ of variable $\omega$ in the action Eq. (18) into two subregions $W$ and $W^{\prime} \backslash W$. The first subregion $W$ results in the normalization factor $\Lambda_{1}$ whereas the subregion $W^{\prime} \backslash W$ results in the factor $\Lambda_{2}$. Despite the nonlinearity term in the action (18) the fields $\phi_{\omega}$ for the different subregions do not mix in these orders in $\tilde{\gamma}$. Therefore $\Lambda$ can be expressed as the product of two path-integrals. The first integral contains the fields $\phi_{\omega}$ for $\omega$ from the subregion $W$ and corresponds to $\Lambda_{1}$. The second one corresponds to $\Lambda_{2}$. Therefore the normalization factor $\Lambda_{1}$ can be expressed in the form of the path-integral (17) where all frequencies are from $W$ subregion.

The details of the factorization of the normalization factor $\Lambda$ in the time domain are presented in the Appendix $B$. The calculation of the averaged $\log \Lambda_{1}$ over PDF $P_{G}\left[\vec{X}_{1}\right]$ is placed in the Appendix C. Here we present only the final result:

$$
I_{P_{G}[X]}=M \log \mathrm{SNR}-M \frac{\widetilde{\gamma}^{2}}{3} g(\widetilde{\beta})+\mathcal{O}\left(\widetilde{\gamma}^{4}\right),
$$

where $g(\widetilde{\beta})$ is the function of dimensionless parameter $\widetilde{\beta}=\beta L W^{2}$, see Eqs. (C32), (C36), (C37):

$$
g(\widetilde{\beta})=4 ! \sum_{n=0}^{\infty} \frac{(-1)^{n} \widetilde{\beta}^{2 n}\left[(4 n+2) !+(1+2 n) !^{2}\right]}{2^{2 n-1}(2 n+4) !(4 n+3) !(1+2 n)^{2}} .
$$

One can check that $g(\widetilde{\beta}=0)=1$. In the case when $\widetilde{\beta} \gg 1$ the asymptotics for the function (48) reads

$$
g(\widetilde{\beta}) \sim \frac{16 \pi}{\widetilde{\beta}}\left(\log \frac{\widetilde{\beta}}{2}+\gamma_{E}-\frac{23}{6}\right)+\mathcal{O}\left(\widetilde{\beta}^{-3 / 2}\right),
$$

where $\gamma_{E} \approx 0.577$ is the Euler constant. The function $g(\widetilde{\beta})$ is plotted in Fig. 1. Note that the result (47) is

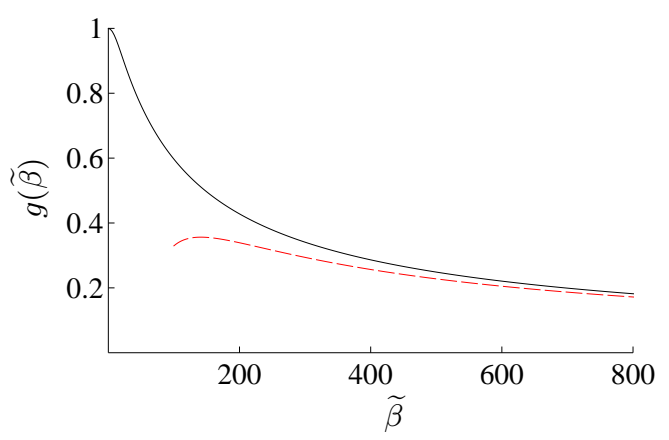

Figure 1: The function $g(\widetilde{\beta})$. The solid black line corresponds to exact expression (48) for $g(\widetilde{\beta})$, the red dashed line corresponds to the asymptotics $(49)$ of $g(\widetilde{\beta})$ at large $\widetilde{\beta}$.

proportional to the number of the meaning channels $M$. 
The reason for that is the definition of the mutual information through the path-integral (1). Usually instead of the mutual information (1) the spectral efficiency is considered as the quantity which does not depend on $M$ :

$$
\begin{aligned}
i_{P[X]}= & \lim _{T \rightarrow \infty} \frac{2 \pi}{T W} I_{P[X]}=\frac{I_{P[X]}}{M}= \\
& \log \mathrm{SNR}-\frac{\widetilde{\gamma}^{2}}{3} g(\widetilde{\beta})+\mathcal{O}\left(\widetilde{\gamma}^{4}\right),
\end{aligned}
$$

where the parameter $T$ is the time duration of the signal. The quantity $i_{P[X]}$ coincides with the per-sample mutual information for the nondispersive case $\beta=0$.

Let us consider the mutual information (43) at zero $\beta$. For the nondispersive case the result for the per-sample mutual information was obtained in Ref. [12]:

$$
i_{P_{G}[X]}^{(\beta=0)}=\log \mathrm{SNR}-\frac{1}{2} \int_{0}^{\infty} d \tau e^{-\tau} \log \left(1+\frac{\tau^{2} \tilde{\gamma}^{2}}{3}\right) .
$$

One can check that at small $\tilde{\gamma}$ the expression (51) reproduces the spectral efficiency $i_{P_{G}[X]}$, see Eq. (50), for $\widetilde{\beta}=0$ :

$$
i_{P_{G}[X]}^{(\beta=0)}=\log \operatorname{SNR}-\frac{\widetilde{\gamma}^{2}}{3}+\mathcal{O}\left(\widetilde{\gamma}^{4}\right) .
$$

Let us estimate the spectral efficiency $i_{P[X]}$ for typical fiber optical links [5]: $\beta=20 \mathrm{ps}^{2} / \mathrm{km}, L=1000 \mathrm{~km}, \gamma=$ $1.31(\mathrm{Wkm})^{-1}, W=100 \mathrm{GHz}, P_{\text {noise }}=Q L W /(2 \pi)=$ $5.3 \times 10^{-4} \mathrm{~mW}$. For these parameters one has $\widetilde{\beta}=$ $\beta L W^{2} \approx 200$, and $g(\widetilde{\beta}) \approx 0.42$. Substituting these parameters to Eq. (50) we obtain

$$
i_{P[X]} \approx \log [\mathrm{SNR}]-7 \times 10^{-8} \times \mathrm{SNR}^{2} .
$$

The behavior of the spectral efficiency for different channels is plotted in Fig. 2. The result (53) is plotted by

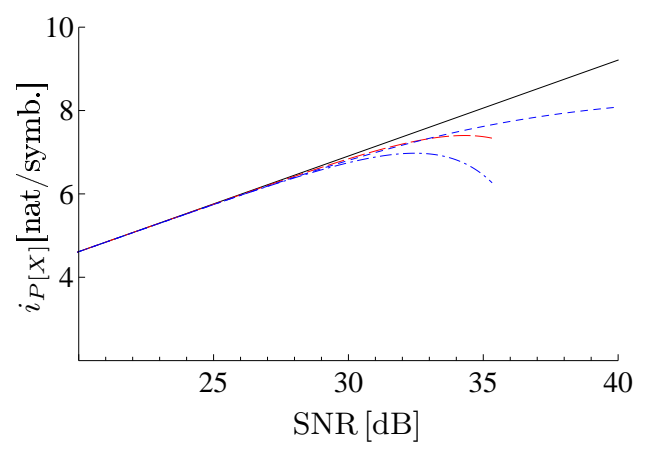

Figure 2: The spectral efficiency $i_{P[X]}$ for different $\widetilde{\beta}$. The solid black line, red long-dashed line, blue dashed, blue dashed-dotted line correspond to $i_{P[X]}$ for a linear channel (Shannon's result), channel for the dispersion $\widetilde{\beta}=200$, see Eq. (53), nondispersive channel Eq. (51), and the expansion (52), respectively.

the red long-dashed line, the exact result for the nondispersive channel (51) and its expansion (52) are plotted by the blue dashed and blue dashed-dotted lines, respectively. The solid black line corresponds to the Shannon's result

$$
i_{P[X]}^{S H}=\log (1+\mathrm{SNR})
$$

for a linear channel $\tilde{\gamma}=0$. One can see that when $\mathrm{SNR} \lesssim 300$ (SNR $\lesssim 25 \mathrm{~dB}$, i.e. $\tilde{\gamma} \lesssim 0.2$ ) the spectral efficiency for different channels is close to the Shannon's result (54). For SNR large than $25 \mathrm{~dB}$ one observes different a behavior for different regimes. The spectral efficiency $i_{P_{G}[X]}^{(\beta=0)}$, see Eq. (51), is the nondecreasing function of the parameter SNR whereas its expansion (52) in $\tilde{\gamma}$ starts decreasing at $\mathrm{SNR} \approx 32 \mathrm{~dB}$. This decreasing is explained by eliminating of higher terms of expansion in $\tilde{\gamma}$. It is interesting that the spectral efficiency for the channel with dispersion for $\widetilde{\beta}=200$, see Eq. (53), is greater than the exact result (51) for zero dispersion in the region $\mathrm{SNR} \lesssim 33 \mathrm{~dB}$, see Fig. 3. One can also see

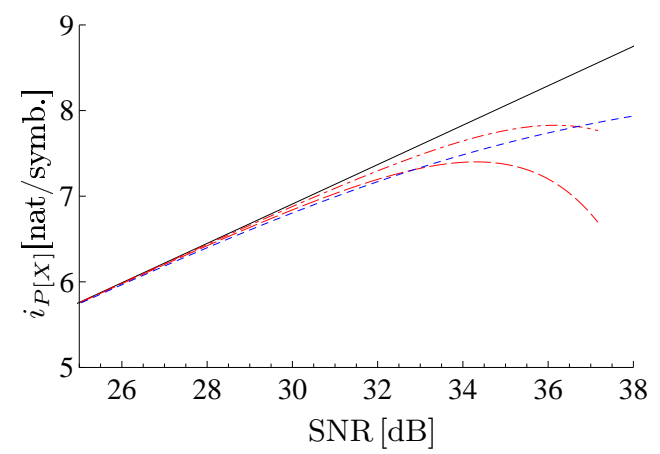

Figure 3: The spectral efficiency $i_{P[X]}$ for different $\widetilde{\beta}$. The solid black line, red long-dashed line, blue dashed, red dasheddotted line correspond to $i_{P[X]}$ for a linear channel (Shannon's result), channel for the dispersion $\widetilde{\beta}=200$, see Eq. (53), nondispersive channel Eq. (51), and the channel for the dispersion $\widetilde{\beta}=800$, see Eq. (53), respectively.

that the spectral efficiency for $\widetilde{\beta}=800$ depicted by the red dashed-dotted line in Fig. 3 is greater than the exact result $(51)$ in the region $\mathrm{SNR} \lesssim 37 \mathrm{~dB}$. Increasing parameter $\widetilde{\beta}$ the first nonlinear correction (see Eq. (50) and asymptotics (49) of the function $g(\widetilde{\beta})$ ) goes to zero as $\tilde{\gamma}^{2} \log (\widetilde{\beta}) / \widetilde{\beta}$. Therefore for larger $\widetilde{\beta}$ the result $(50)$ is closer to Shannon's result (54) than the result (51) in wider region in SNR.

Let us consider the applicability region of our result (50). To calculate the spectral efficiency (50) we have used the perturbative expansion of the normalization factor $\Lambda_{1}$ in the parameter $\tilde{\gamma}$. Formally, the applicability region of our result (50) is defined by the conditions that the found correction must be much less than the main term (log SNR in our case), and also the next correction of order of $\tilde{\gamma}^{4}$ must be much less than the correction of order of $\tilde{\gamma}^{2}$. We can estimate the next correction in the dispersive case using the next correction in $\widetilde{\gamma}$ for the nondispersive case. Performing an expansion in 
Eq. (51) in $\tilde{\gamma}$ we derive that the next correction has the form $+2 \tilde{\gamma}^{4} / 3$. However, for the dispersive case instead of $\tilde{\gamma}^{2}$ we have an additional suppression factor $g(\widetilde{\beta}) \leq 1$. And there is an indication that at large $\widetilde{\beta}$, the effective parameter of the perturbative series is $\tilde{\gamma}^{2} \log (\widetilde{\beta}) / \widetilde{\beta}$ rather than $\tilde{\gamma}^{2}$ : see Eq. (49). Physically it means that in the case of large $\widetilde{\beta}$ the dispersion leads to signal spreading in time domain. It results in the amplitude decreasing and thereby decreasing of the nonlinear term in the equation (7). Therefore the effective expansion parameter should be suppressed at large $\widetilde{\beta}$. And we can estimate the next correction in the dispersive case as $\sim+\left(g(\widetilde{\beta}) \tilde{\gamma}^{2}\right)^{2}$. Therefore by increasing the parameter $\widetilde{\beta}$ we increase the SNR region of applicability of our result (50). Providing the validity of the indication about the parameter of the perturbative series our result (53) for $\widetilde{\beta}=200$ is applicable in the region $\mathrm{SNR} \lesssim 30 d B$, whereas for $\widetilde{\beta}=800$ the region is $\mathrm{SNR} \lesssim 35 d B$.

\section{CONCLUSION}

We have derived the analytical expression for the mutual information $I_{P[X]}$ of the channel modelled by the nonlinear Schrödinger equation with the additive Gaussian noise at large SNR. We have calculated analytically the first nonlinear correction to the mutual information in the nonlinearity parameter $\tilde{\gamma}=\gamma L P_{\text {ave }}$. We have analyzed the obtained result for different values of the dispersion parameter $\beta$, and we have shown that there is the region in parameter SNR where the spectral efficiency (50) for nonzero dispersion channel is greater than the exact result (51) for the nondispersive channel. We have also shown that our result for the spectral efficiency (50) for nonzero dispersion approaches the expression (52) derived in Ref. [12] in the case when $\beta$ tends to zero.

\section{Acknowledgments}

The general expressions for the entropies and the mutual information have been obtained with the support of the Russian Science Foundation (RSF) (grant No. 1611-10133). Part of the work (perturbative calculation of the mutual information) was supported by the Russian Foundation for Basic Research (RFBR), Grant No. 16-31-60031/15. A. V. Reznichenko thanks the President program (CП-2415.2015.2) for support. The work of S. K. Turitsyn was supported by the grant of the Ministry of Education and Science of the Russian Federation (agreement No. 14.B25.31.0003) and the EPSRC project UNLOC.

\section{Appendix A: CLASSICAL SOLUTION OF THE EULER-LAGRANGE EQUATION $\delta S[\Psi]=0$}

In Ref.[19] the following representation for the conditional probability $P[Y \mid X]$ was obtained:

$$
\begin{aligned}
& P[Y \mid X]=\Lambda \exp \left\{-\frac{S[\Psi]}{Q}\right\}, \\
& \Lambda=\int_{\phi(z=0)=0}^{\phi(z=L)=0} \mathcal{D} \phi \exp \left\{-\frac{1}{Q}(S[\Psi+\phi]-S[\Psi])\right\},
\end{aligned}
$$

where the action $S[\psi]$ in (A1) reads in the time domain

$$
\begin{aligned}
& S[\psi]=\int_{0}^{L} d z \int_{T} d t|\mathcal{L}[\psi(z, t)]|^{2}, \\
& \mathcal{L}[\psi]=\partial_{z} \psi+i \beta \partial_{t}^{2} \psi-i \gamma \psi|\psi|^{2} .
\end{aligned}
$$

Here $T$ is a time interval containing both signals $X(t)$ and $Y(t)$. In the following discretization scheme we use the relations between $T$ and discretization intervals in the time domain ( $\delta_{t}$ for the "dense" time grid with $M^{\prime}$ intervals and $\tilde{\delta}_{t}$ for the "coarse" time sub-grid with $M$ intervals) and in the frequency domain $\left(\delta_{\omega}\right)$ :

$$
T=\frac{1}{\delta_{\omega}}=M^{\prime} \delta_{t}=M \tilde{\delta}_{t}=\frac{2 \pi}{W} M=\frac{2 \pi}{W^{\prime}} M^{\prime} .
$$

The function $\Psi$ in Eq. (A1) is the solution (referred to as "the classical solution") of the Euler-Lagrange equation $\delta S[\Psi]=0$ with the boundary conditions: $\Psi(0)=$ $X, \quad \Psi(L)=Y$. In the time domain this equation for $\Psi(z, t)$ has a notedly simple form

$$
\begin{aligned}
& \left(\partial_{z}+i \beta \partial_{t}^{2}-2 i \gamma|\Psi(z, t)|^{2}\right) \mathcal{L}[\Psi(z, t)]+ \\
& i \gamma \Psi^{2}(z, t) \overline{\mathcal{L}[\Psi(z, t)]}=0, \\
& \mathcal{L}[\Psi(z, t)]=\left(\partial_{z}+i \beta \partial_{t}^{2}-i \gamma|\Psi(z, t)|^{2}\right) \Psi(z, t),
\end{aligned}
$$

and the function $\Psi(z, t)$ obeys the boundary conditions: $\Psi(0, t)=X(t), \Psi(L, t)=Y(t)$. The bar in Eq. (A4) and hereafter means complex conjugation.

It is convenient to introduce the function $\Phi(z, t)$ which is the solution of the nonlinear Schrödinger equation (NLSE)

$$
\partial_{z} \Phi(z, t)+i \beta \partial_{t}^{2} \Phi(z, t)-i \gamma \Phi(z, t)|\Phi(z, t)|^{2}=0
$$

with the boundary condition $\Phi(0, t)=X(t)$. One can see that the equation (A5) can be written as

$$
\mathcal{L}[\Phi(z, t)]=0 .
$$

Therefore it is obvious that the function $\Phi(z, t)$ obeys the equation Eq. (A4) and the boundary condition at $z=0$, but it does not obey the boundary condition at $z=L$. It globally minimizes the action as well: $S[\Phi(z, t)]=0$. Since we imply that the noise power is much less than 
the signal power we can present the solution of Eq. (A4) in the form

$$
\Psi(z, t)=\Phi(z, t)+\varkappa(z, t)
$$

where the function $\varkappa(z, t)$ is of order of $\sqrt{Q}$ for unsuppressed configurations $\Psi(z, t)$. Therefore we substitute the function $\Psi$ in the form (A7) to the Eq. (A4), then linearizing Eq. (A4) in $\varkappa(z, t)$ we obtain the following linear problem on $\varkappa(z, t)$ :

$$
\begin{aligned}
& \left(\partial_{z}+i \beta \partial_{t}^{2}-2 i \gamma|\Phi(z, t)|^{2}\right) l[\varkappa]+i \gamma \Phi^{2}(z, t) \overline{l[\varkappa]}=0 \\
& l[\varkappa]=\left(\partial_{z}+i \beta \partial_{t}^{2}\right) \varkappa(z, t)- \\
& i \gamma\left(2 \varkappa(z, t)|\Phi(z, t)|^{2}+\overline{\varkappa(z, t)} \Phi^{2}(z, t)\right),
\end{aligned}
$$

with the boundary conditions

$$
\begin{aligned}
& \varkappa(z=0, t)=0, \\
& \varkappa(z=L, t)=Y(t)-\Phi(L, t) \equiv \delta Y(t) .
\end{aligned}
$$

Being rewritten explicitly Eq. (A8) has the following form

$$
\begin{aligned}
& {\left[\left(\partial_{z}+i \beta \partial_{t}^{2}\right)^{2}-4 i \gamma|\Phi|^{2}\left(\partial_{z}+i \beta \partial_{t}^{2}\right)-5 \gamma^{2}|\Phi|^{4}+\right.} \\
& \left.4 \beta \gamma\left(\Phi \frac{\partial^{2} \bar{\Phi}}{\partial t^{2}}+\left|\frac{\partial \Phi}{\partial t}\right|^{2}+\frac{\partial|\Phi|^{2}}{\partial t} \partial_{t}\right)\right] \varkappa+ \\
& 2 \gamma\left[-\gamma \Phi^{2}|\Phi|^{2}+\beta \Phi^{2} \partial_{t}^{2}+\beta \frac{\partial \Phi^{2}}{\partial t} \partial_{t}+\right. \\
& \left.\beta\left(\frac{\partial \Phi}{\partial t}\right)^{2}\right] \bar{\varkappa}=0
\end{aligned}
$$

where we have used that $\Phi(z, t)$ is the solution of Eq. (A5). In the frequency domain the Euler-Lagrange equation $\delta S[\Psi]=0$ for the solution $\Psi_{\omega}(z)$ has the form [19]:

$$
\begin{aligned}
& \left(\partial_{z}-i \beta \omega^{2}\right)^{2} \Psi_{\omega}(z)-i \gamma \int \frac{d \omega_{1} d \omega_{2} d \omega_{3}}{(2 \pi)^{2}} \delta\left(\omega+\omega_{3}-\omega_{1}-\omega_{2}\right)\left\{4 \Psi_{\omega_{2}}(z) \bar{\Psi}_{\omega_{3}}(z)\left(\partial_{z}-i \beta \omega_{1}^{2}\right) \Psi_{\omega_{1}}(z)-\right. \\
& \left.i \beta\left(\omega^{2}-\omega_{1}^{2}-\omega_{2}^{2}+\omega_{3}^{2}\right) \Psi_{\omega_{1}}(z) \Psi_{\omega_{2}}(z) \bar{\Psi}_{\omega_{3}}(z)\right\}- \\
& 3 \gamma^{2} \int \frac{d \omega_{1} d \omega_{2} d \omega_{4} d \omega_{5} d \omega_{6}}{(2 \pi)^{4}} \delta\left(\omega_{1}+\omega_{2}+\omega_{4}-\omega_{5}-\omega_{6}-\omega\right) \Psi_{\omega_{1}}(z) \Psi_{\omega_{2}}(z) \Psi_{\omega_{4}}(z) \bar{\Psi}_{\omega_{5}}(z) \bar{\Psi}_{\omega_{6}}(z)=0,
\end{aligned}
$$

with the boundary conditions: $\Psi_{\omega}(0)=X(\omega), \Psi_{\omega}(L)=Y(\omega)$. The equation (A10) for the function $\varkappa$ can be rewritten in the frequency domain as follows:

$$
\begin{aligned}
& \left(\partial_{z}-i \beta \omega^{2}\right)^{2} \varkappa_{\omega}(z)-i \gamma \int \frac{d \omega_{1} d \omega_{2} d \omega_{3}}{(2 \pi)^{2}} \delta\left(\omega+\omega_{3}-\omega_{1}-\omega_{2}\right)\left\{4 \Phi_{\omega_{2}}(z) \bar{\Phi}_{\omega_{3}}(z)\left(\partial_{z}-i \beta \omega_{1}^{2}\right) \varkappa_{\omega_{1}}(z)-\right. \\
& \left.i \beta\left(\omega^{2}-\omega_{1}^{2}-\omega_{2}^{2}+\omega_{3}^{2}\right) \Phi_{\omega_{2}}(z)\left[2 \varkappa_{\omega_{1}}(z) \bar{\Phi}_{\omega_{3}}(z)+\bar{\varkappa}_{\omega_{3}}(z) \Phi_{\omega_{1}}(z)\right]\right\}- \\
& \gamma^{2} \int \frac{d \omega_{1} d \omega_{2} d \omega_{4} d \omega_{5} d \omega_{6}}{(2 \pi)^{4}} \delta\left(\omega_{1}+\omega_{2}+\omega_{4}-\omega_{5}-\omega_{6}-\omega\right) \Phi_{\omega_{2}}(z) \Phi_{\omega_{4}}(z) \bar{\Phi}_{\omega_{5}}(z) \times \\
& \times\left[5 \varkappa_{\omega_{1}}(z) \bar{\Phi}_{\omega_{6}}(z)+2 \bar{\varkappa}_{\omega_{6}}(z) \Phi_{\omega_{1}}(z)\right]=0 .
\end{aligned}
$$

Of course, Eq. (A12) can be obtained from Eq. (A11) by the linearization procedure using Eq. (A5) for the function $\Phi_{\omega}(z)$.

In our model there are two grids both in frequency and time domains. In the frequency domain $W$ we have $M$ grid points corresponding to the meaning complex channels and $M^{\prime}-M$ points in the domain $W^{\prime} \backslash W$ corresponding to the channels with zero input signal $X(\omega)$. These grids in the frequency domain relate with the coarse and dense grids in the time domain. Grid points in the coarse time grid are separated by intervals $\delta_{t}=T / M=2 \pi / W$ and carry information about $M$ meaning channels. The dense time grid contains $M^{\prime}$ points separated by intervals $\delta_{t}=T / M^{\prime}=2 \pi / W^{\prime}$, see Eq. (A3). The signals
$X\left(t_{k}\right)$ in $M^{\prime}-M$ remnant points are uniquely defined by the signals on the coarse grid. Therefore to obtain Eqs. (A11) and (A12) in our frequency discretization scheme we should perform the following substitutions:

$$
\begin{aligned}
& \partial_{t}^{2} \Phi(z, t) \rightarrow-\Omega_{k}^{2} \Phi_{\omega_{k}}(z) \\
& \delta\left(\omega_{i_{1}}+\omega_{i_{2}}+\ldots\right) \rightarrow \frac{\Delta_{\left(M^{\prime}\right)}\left(i_{1}+i_{2}+\ldots\right)}{2 \pi \delta_{\omega}} \\
& \int \frac{d \omega}{2 \pi} \ldots \rightarrow \delta_{\omega} \sum_{j^{\prime}=0}^{M^{\prime}-1}
\end{aligned}
$$


where we use the notations:

$$
\begin{aligned}
& \omega_{n^{\prime}}=-W^{\prime} / 2+2 \pi \delta_{\omega} n^{\prime}, \\
& \Omega_{n^{\prime}}=2 \sin \left[\pi \frac{n^{\prime}}{M^{\prime}}\right] \frac{M^{\prime}}{T}=2 M^{\prime} \delta_{\omega} \sin \left[\pi \frac{n^{\prime}}{M^{\prime}}\right]
\end{aligned}
$$

for $n^{\prime}=0,1, \ldots, M^{\prime}-1$, and in Eq. (A13) we use

$$
\Delta_{(M)}(k)=\frac{1}{M} \sum_{n=0}^{M-1} e^{-2 \pi i \frac{n}{M} k}=\sum_{m=-\infty}^{\infty} \delta_{k, m M} .
$$

\section{Appendix B: FACTORIZATION OF PRE-EXPONENTIAL FACTOR $\Lambda$}

Let us consider the separation of different scales in the conditional PDF $P[Y \mid X]$ when PDF $P[Y \mid X]$ is considered under an integral over $X$ together with the initial signal PDF $P[X]$ which has the following form in the frequency domain:

$$
P[\vec{X}]=P_{X}^{(M)}\left[\vec{X}_{1}\right] \delta\left(\vec{X}_{2}\right)
$$

where $\delta\left(\vec{X}_{2}\right)$ means $2\left(M^{\prime}-M\right)$-dimensional deltafunction corresponding $M^{\prime}-M$ complex remnant channels. We remind that the vector notations (introduced in the main text of our manuscript, see the beginning of the Section III) $\vec{X}=\vec{X}_{1} \oplus \vec{X}_{2}$ relate to the frequency domain: $\vec{X}_{1}$ is $2 M$-dimensional vector corresponding to $M$ meaning complex channels in the frequency domain $W$, whereas $\vec{X}_{2}$ is $2\left(M^{\prime}-M\right)$-dimensional vector corresponding to remnant $M^{\prime}-M$ complex channels in the frequency domain $W^{\prime} \backslash W$. Now we are going to demonstrate the factorization property of $P[Y \mid X]$ in the time domain.

In the leading order in $1 / \mathrm{SNR}$ the conditional PDF $P[Y \mid X]$ can be obtained by substituting $\Psi$ in the form $\Psi=\Phi+\varkappa$ to the Eq. (A1). After obvious transformations, see Section III, we obtain:

$$
P[Y \mid X] \approx \Lambda[X] e^{-S_{2}[\varkappa] / Q}
$$

where the action $S_{2}[\phi]$ is quadratic functional in $\phi$ and it reads, see Eq. (23),

$$
S_{2}[\phi]=\delta_{t} \sum_{k=0}^{M^{\prime}-1} \Delta \sum_{n=1}^{N-1} \mathcal{L}_{e f f}\left[\phi\left(z_{n}, t_{k}\right)\right],
$$

where $\delta_{t}=T / M^{\prime}$ is the discretization parameter in the time domain: $t_{k}=k \delta_{t}, k=0,1, \ldots, M^{\prime}-1$. In Eq. (B3) $\Delta=L / N$ is the distance discretization parameter: $z_{n}=$ $n \Delta, n=1,2, \ldots, N-1$. In the action (B3) we have introduced the "Lagrangian":

$$
\begin{aligned}
& \mathcal{L}_{\text {eff }}[\varkappa]=\mid \partial_{z} \varkappa\left(z_{n}, t_{k}\right)+i \beta \partial_{t}^{2} \varkappa\left(z_{n}, t_{k}\right)- \\
& \left.i \gamma\left(2 \varkappa\left(z_{n}, t_{k}\right)\left|\Phi\left(z_{n}, t_{k}\right)\right|^{2}+\bar{\varkappa}\left(z_{n}, t_{k}\right) \Phi^{2}\left(z_{n}, t_{k}\right)\right)\right|^{2} .
\end{aligned}
$$

Here derivatives should be regarded as difference derivatives in our discretization scheme. The function $\varkappa(z, t)$ in the exponent (B2) is the solution of the EulerLagrange equation $-\delta \mathcal{L}_{\text {eff }}[\varkappa] / \delta \bar{\varkappa}=0$ which coincides with Eq. (A10) with the boundary conditions $\varkappa(z=$ $0, t)=0, \varkappa(z=L, t)=Y(t)-\Phi(L, t)$. Here $\Phi(L, t)=$ $\hat{\mathrm{L}} X(t)$ is the solution of NLSE with the zero noise and with the input boundary condition, see Eq. (A6). The normalization factor $\Lambda[X]$ has the form

$$
\Lambda[X]=\int_{\phi(0, t)=0}^{\phi(L, t)=0} \mathcal{D} \phi e^{-\frac{\delta_{t}}{Q} \sum_{k=0}^{M^{\prime}-1} \Delta \sum_{n=1}^{N-1} \mathcal{L}_{e f f}\left[\phi\left(z_{n}, t_{k}\right)\right]} .
$$

Note that the sum in Eq. (B3) is performed over the dense time grid. To demonstrate the factorization we have to separate the scales in the action into the coarse and dense parts. In other words, we have to separate the summation over $M$ meaning channels and $M^{\prime}-M$ remnant channels. The scale separation procedure in some sense is similar to Wilson's renormalization procedure for the Lagrangian $\mathcal{L}_{\text {eff }}[\varkappa]$, see [25]. But in our approximation the Lagrangian (B4) is quadratic functional in $\varkappa$ that is why there are no corrections to the effective action when we perform integration over remnant $2\left(M^{\prime}-M\right)$ degrees of freedom $\varkappa\left(z, t_{k}\right)$ where $t_{k}$ runs through values only on the dense grid without the coarse sub-grid. Let us demonstrate this fact.

First we perform the separation of variables:

$$
\begin{aligned}
& \varkappa\left(z, t_{k}\right)=\varkappa^{(c)}\left(z, t_{k}\right)+\varkappa^{(d)}\left(z, t_{k}\right), \\
& Y\left(t_{k}\right)=Y^{(c)}\left(t_{k}\right)+Y^{(d)}\left(t_{k}\right),
\end{aligned}
$$

where $\varkappa^{(c)}\left(z, t_{k}\right)$, or $Y^{(c)}\left(t_{k}\right)$, is completely defined only by the values $\varkappa^{(c)}\left(z, T_{\tilde{i}}\right)$, or $Y^{(c)}\left(T_{\tilde{i}}\right)$, on the coarse time grid $T_{\tilde{i}}=\tilde{i} \tilde{\delta}_{t}, \tilde{i}=0,1, \ldots, M-1$. Here and below superscript "(c)" means "coarse" variable. In other words, the function $\varkappa^{(c)}\left(z, t_{k}\right)$ evaluated in all grid points is the interpolation of some order (i.e. the interpolating polynomial degree) $N_{0}>2$ calculated on the base of values $\varkappa^{(c)}\left(z, T_{\tilde{i}}\right)$ of the coarse time grid. The function $\varkappa^{(c)}\left(z, t_{k}\right)$ coincides with $\varkappa\left(z, T_{\tilde{i}}\right)$ when $t_{k}$ falls on the coarse time grid $T_{\tilde{i}}$ (i.e. $k=\left[\tilde{i} M^{\prime} / M\right]$, $\tilde{i}=0,1, \ldots, M-1)$, i.e. $\varkappa^{(d)}\left(z, t_{k}\right)=0$ on the coarse grid. In other grid points of the dense grid the function $\varkappa^{(c)}\left(z, t_{k}\right)$ smoothly interpolates the values of $\varkappa\left(z, t_{k}\right)$ with interpolation order $N_{0}>2: \varkappa^{(d)}=\mathcal{O}\left(\tilde{\delta}_{t}^{N_{0}}\right)$ and $\partial_{t}^{2} \varkappa^{(d)}\left(z, t_{k}\right)=\mathcal{O}\left(\tilde{\delta}_{t}^{N_{0}-2}\right)$, where we have used that $\partial_{t}^{2} \varkappa\left(z, t_{k}\right)=\partial_{t}^{2} \varkappa^{(c)}\left(z, t_{k}\right)+\partial_{t}^{2} \varkappa^{(d)}\left(z, t_{k}\right)$, here the derivatives are assumed as the difference derivatives on the dense grid. The boundary conditions are as follows:

$$
\begin{aligned}
& \varkappa^{(c)}\left(0, t_{k}\right)=0, \\
& \varkappa^{(c)}\left(L, t_{k}\right)=Y^{(c)}\left(t_{k}\right)-\Phi^{(c)}\left(L, t_{k}\right), \\
& \varkappa^{(d)}\left(0, t_{k}\right)=0, \\
& \varkappa^{(d)}\left(L, t_{k}\right)=Y^{(d)}\left(t_{k}\right)-\Phi^{(d)}\left(L, t_{k}\right),
\end{aligned}
$$


where we have used that $\Phi\left(L, t_{k}\right)$ has the "coarse" and "dense" parts as well:

$$
\begin{aligned}
& \Phi\left(z, t_{k}\right)=\Phi^{(c)}\left(z, t_{k}\right)+\Phi^{(d)}\left(z, t_{k}\right), \\
& \Phi^{(d)}\left(z, t_{k}\right)=\mathcal{O}\left(\tilde{\delta}_{t}^{N_{0}}\right) .
\end{aligned}
$$

Note that if we consider (B2) under the integral over $\mathcal{D} X$ with the initial signal PDF (B1), then the function
$\Phi\left(z, t_{k}\right)$ is the (nonlinear) function of the input signal only on the coarse time grid $X\left(T_{\tilde{i}}\right)$. This means that the "dense" part $\Phi^{(d)}\left(z, t_{k}\right)=\mathcal{O}\left(\tilde{\delta}_{t}^{N_{0}}\right)$ is always small for sufficiently large $M$.

Now we insert the representation (B6) in the action (B3). The action fractionizes into three parts

$$
\begin{aligned}
& S_{2}[\varkappa]=\delta_{t} \sum_{k=0}^{M^{\prime}-1} \Delta \sum_{n=1}^{N-1} \mathcal{L}_{e f f}\left[\varkappa\left(z_{n}, t_{k}\right)\right]=\delta_{t} \sum_{k=0}^{M^{\prime}-1} \Delta \sum_{n=1}^{N-1} \mathcal{L}_{e f f}\left[\varkappa^{(c)}\left(z_{n}, t_{k}\right)\right]+\delta_{t} \sum_{k=0}^{M^{\prime}-1} \Delta \sum_{n=1}^{N-1} \mathcal{L}_{\text {eff }}\left[\varkappa^{(d)}\left(z_{n}, t_{k}\right)\right]+ \\
& \delta_{t} \sum_{k=0}^{M^{\prime}-1} \Delta \sum_{n=1}^{N-1} \mathcal{L}_{\text {int }}\left[\varkappa^{(c)}\left(z_{n}, t_{k}\right), \varkappa^{(d)}\left(z_{n}, t_{k}\right)\right]
\end{aligned}
$$

where the third part with interaction of "coarse" $\left(\varkappa^{(c)}\right)$ and "dense" $\left(\varkappa^{(d)}\right)$ degrees of freedom contains Lagrangian

$$
\begin{aligned}
& \mathcal{L}_{\text {int }}\left[\varkappa^{(c)}\left(z, t_{k}\right), \varkappa^{(d)}\left(z, t_{k}\right)\right]= \\
& \left(\partial_{z} \varkappa^{(c)}\left(z, t_{k}\right)+i \beta \partial_{t}^{2} \varkappa^{(c)}\left(z, t_{k}\right)-i \gamma\left(2 \varkappa^{(c)}\left(z, t_{k}\right)\left|\Phi\left(z, t_{k}\right)\right|^{2}+\bar{\varkappa}^{(c)}\left(z, t_{k}\right) \Phi^{2}\left(z, t_{k}\right)\right)\right) \times \\
& \left(\partial_{z} \bar{\varkappa}^{(d)}\left(z, t_{k}\right)-i \beta \partial_{t}^{2} \bar{\varkappa}^{(d)}\left(z, t_{k}\right)+i \gamma\left(2 \bar{\varkappa}^{(d)}\left(z, t_{k}\right)\left|\Phi\left(z, t_{k}\right)\right|^{2}+\varkappa^{(d)}\left(z, t_{k}\right) \bar{\Phi}^{2}\left(z, t_{k}\right)\right)\right)+c . c .
\end{aligned}
$$

Here "c.c." means the same complex conjugated term.

The first part in the r.h.s. of Eq. (B9) can be simplified as follows:

$$
\begin{aligned}
& \delta_{t} \sum_{k=0}^{M^{\prime}-1} \Delta \sum_{n=1}^{N-1} \mathcal{L}_{\text {eff }}\left[\varkappa^{(c)}\left(z_{n}, t_{k}\right)\right]= \\
& \tilde{\delta}_{t} \sum_{\tilde{i}=0}^{M-1} \Delta \sum_{n=1}^{N-1} \mathcal{L}_{\text {eff }}\left[\phi\left(z_{n}, T_{\tilde{i}}\right)\right]\left(1+\mathcal{O}\left(\tilde{\delta}_{t}^{N_{0}-2}\right)\right)
\end{aligned}
$$

where $\tilde{\delta}_{t}=\frac{2 \pi}{W}=\delta_{t}\left[M^{\prime} / M\right]$ is the grid spacing of the coarse time grid. Here we have replaced every term under the sum over the dense grid with its average value on the coarse grid. The accuracy in Eq. (B11) is governed by interpolation order of the second derivative in time.

The third part in the r.h.s. of Eq. (B9) can be integrated (summed) over $z$ by part resulting in the following expression:

$$
\begin{aligned}
& \delta_{t} \sum_{k=0}^{M^{\prime}-1} \Delta \sum_{n=1}^{N-1} \mathcal{L}_{\text {int }}\left[\varkappa^{(c)}\left(z_{n}, t_{k}\right), \varkappa^{(d)}\left(z_{n}, t_{k}\right)\right]= \\
& \delta_{t} \sum_{k=0}^{M^{\prime}-1} \Delta \sum_{n=1}^{N-1}\left(\bar{\varkappa}^{(d)}\left(z_{n}, t_{k}\right) \frac{\delta \mathcal{L}_{\text {eff }}\left[\varkappa^{(c)}\right]}{\delta \varkappa}+h . c .\right)+ \\
& S_{\text {surf }},
\end{aligned}
$$

where the variation $-\delta \mathcal{L}_{\text {eff }}\left[\varkappa^{(c)}\right] / \delta \varkappa$ is linear in $\varkappa^{(c)}$, and it represents the 1.h.s. of the Euler-Lagrange equation (A10), i.e. for the function $\varkappa^{(c)}$ we obtain $\delta \mathcal{L}_{\text {eff }}\left[\varkappa^{(c)}\right] / \delta \varkappa=\mathcal{O}\left(\tilde{\delta}_{t}^{N_{0}-2}\right)$, i.e. it is always small. The term $S_{\text {surf }}$ results from the surface term in integration by part over $z$ in Eq. (B13), and taking into account the boundary conditions (B7) it reads

$$
\begin{aligned}
& S_{\text {surf }}=\delta_{t} \sum_{k=0}^{M^{\prime}-1}\left[\bar{Y}^{(d)}\left(t_{k}\right)-\bar{\Phi}^{(d)}\left(L, t_{k}\right)\right] \times \\
& \left(\partial_{z} \varkappa^{(c)}\left(L, t_{k}\right)+i \beta \partial_{t}^{2} \varkappa^{(c)}\left(L, t_{k}\right)-\right. \\
& \left.i \gamma\left(2 \varkappa^{(c)}\left(L, t_{k}\right)\left|\Phi\left(L, t_{k}\right)\right|^{2}+\bar{\varkappa}^{(c)}\left(L, t_{k}\right) \Phi^{2}\left(L, t_{k}\right)\right)\right)+ \\
& \text { c.c. }
\end{aligned}
$$

We can omit the surface term (B14), since it is linear both in the "coarse" and "dense" variables, but they are orthogonal when integrating over $t$ (they have not intersecting supports in the frequency domain). It is obvious for the first two terms in the parentheses in Eq. (B14). The last terms containing $\Phi\left(L, t_{k}\right)$ are "coarse" variables as well: we can replace $\Phi\left(L, t_{k}\right)$ with $\Phi^{(c)}\left(L, t_{k}\right)$ with the interpolation accuracy $\mathcal{O}\left(\tilde{\delta}_{t}^{N_{0}}\right)$ and then replace $\Phi^{(c)}\left(L, t_{k}\right)$ with $Y^{(c)}\left(t_{k}\right)$ with the accuracy $\mathcal{O}(\sqrt{Q})$ (we remind that $\varkappa$ in Eq. (B7) is of order of $\sqrt{Q})$. Then we can replace $Y^{(c)}\left(t_{k}\right)$ with the constants inside the whole interval of an coarse space with the interpolation accuracy $\mathcal{O}\left(\tilde{\delta}_{t}\right)$ and now use the orthogonality of the "coarse" and "dense" variables.

To summarize, with the accuracy of our interpolation $\mathcal{O}\left(\tilde{\delta}_{t}\right)=\mathcal{O}(1 / M)$ we can omit the interaction term (B13), 
and our action fractionizes into "coarse" and "dense" parts:

$$
S_{2}[\varkappa] \approx S_{2}\left[\varkappa^{(c)}\right]+S_{2}\left[\varkappa^{(d)}\right]
$$

where we have separated "coarse" and "dense" degrees of freedom: $S_{2}\left[\varkappa^{(c)}\right]$ depends on $Y^{(c)}\left(t_{j}\right)$, and $S_{2}\left[\varkappa^{(d)}\right]$ depends on $Y^{(d)}\left(t_{j}\right)$ only. Both actions are expressed through the same Lagrangian (B4) and are represented as the quadratic forms. The coefficients of these quadratic forms depend on input signal $X$ only.

The factorization of $\Lambda$, see Eq. (38), can be shown using the normalization condition:

$$
1=\int \mathcal{D} Y P[Y \mid X]=\Lambda \int \mathcal{D} Y e^{-S_{2}[\varkappa] / Q},
$$

where we have used that $\Lambda$ does not depend on $Y$ in the leading order in 1/SNR. Taking into account (B16) and (B15) we obtain

$$
\begin{aligned}
& \Lambda^{-1}=\int \mathcal{D} Y \exp \left\{-\frac{S_{2}\left[\varkappa^{(c)}\right]}{Q}-\frac{S_{2}\left[\varkappa^{(d)}\right]}{Q}\right\}= \\
& \int \mathcal{D} Y^{(c)} \exp \left\{-\frac{S_{2}\left[\varkappa^{(c)}\right]}{Q}\right\} \mathcal{D} Y^{(d)} \exp \left\{-\frac{S_{2}\left[\varkappa^{(d)}\right]}{Q}\right\}= \\
& \Lambda_{1}^{-1} \times \Lambda_{2}^{-1},
\end{aligned}
$$

or

$$
\Lambda=\Lambda_{1} \times \Lambda_{2}
$$

Here the normalization factor $\Lambda_{1}$ depends on the input signal $X$ on the coarse grid only and reads

$$
\begin{aligned}
& \Lambda_{1}=\int_{\phi(0, t)=0}^{\phi(L, t)=0}[\mathcal{D} \phi(z, t)]_{M} \exp \left\{-\frac{\tilde{\delta}_{t}}{Q} \sum_{\tilde{i}=0}^{M-1} \Delta \sum_{n=1}^{N-1} \mathcal{L}_{e f f}\left[\phi\left(z_{n}, T_{\tilde{i}}\right)\right]\right\} \\
& \mathcal{L}_{\text {eff }}\left[\phi\left(z_{n}, T_{\tilde{i}}\right)\right]=\mid \partial_{z} \phi\left(z_{n}, T_{\tilde{i}}\right)+i \beta \partial_{t}^{2} \phi\left(z_{n}, T_{\tilde{i}}\right)- \\
& \left.i \gamma\left(2 \phi\left(z_{n}, T_{\tilde{i}}\right)\left|\Phi\left(z_{n}, T_{\tilde{i}}\right)\right|^{2}+\bar{\phi}\left(z_{n}, T_{\tilde{i}}\right) \Phi^{2}\left(z_{n}, T_{\tilde{i}}\right)\right)\right|^{2}
\end{aligned}
$$

The measure $[\mathcal{D} \phi(z, t)]_{M}$ is defined as

$$
\begin{aligned}
& {[\mathcal{D} \phi(z, t)]_{M}=\lim _{\tilde{\delta}_{t} \rightarrow 0} \lim _{\Delta \rightarrow 0}\left(\frac{\tilde{\delta}_{t}}{\Delta \pi Q}\right)^{M} \times} \\
& \prod_{\tilde{j}=0}^{M-1} \prod_{i=1}^{N-1}\left\{\frac{\tilde{\delta}_{t}}{\Delta \pi Q} d \operatorname{Re} \phi\left(z_{i}, T_{\tilde{j}}\right) d \operatorname{Im} \phi\left(z_{i}, T_{\tilde{j}}\right)\right\}
\end{aligned}
$$

In Eq. (B18) the normalization factor $\Lambda_{1}$ corresponds to $M$ meaning complex channels, and $\Lambda_{2}$ corresponds to $M^{\prime}-M$ complex remnant channels. In this demonstration we have used that the quantity $P[Y \mid X]$ is considered under the integral over $\mathcal{D} X$ with the initial signal PDF $P[X]$, see Eq. (B1). The accuracy of our factorization is at least $\mathcal{O}\left(\tilde{\delta}_{t}\right)=\mathcal{O}(1 / M)$.
For illustration of the factorization (B18) let us consider the factorization property for the conditional PDF $P[Y \mid X]$ and for $\Lambda$ in two cases: a linear channel with nonzero dispersion and a nonlinear nondispersive channel [12].

The linear channel $(\gamma=0)$ with dispersion has this exact property notedly simple in the frequency domain, see e.g. [19]:

$$
\begin{aligned}
& P[Y \mid X]=P^{(M)}[Y \mid X] \times P^{\left(M^{\prime}-M\right)}[Y \mid X]=\left(\frac{\delta_{\omega}}{\pi Q L}\right)^{M^{\prime}} \times \\
& \exp \left\{-\frac{\delta_{\omega}}{Q L} \sum_{k^{\prime}=0}^{M^{\prime}-1} \mid Y\left(\omega_{k^{\prime}}\right) e^{\left.-i \beta L \Omega_{k^{\prime}}^{2}-\left.X\left(\omega_{k^{\prime}}\right)\right|^{2}\right\} .}(\mathrm{B} 21)\right.
\end{aligned}
$$

In the linear case the same factorization is valid for the normalization factor acquiring the trivial form: $\Lambda=$ $\left(\delta_{\omega} /(\pi Q L)\right)^{M^{\prime}}=\Lambda_{1} \times \Lambda_{2}, \Lambda_{1}=\left(\delta_{\omega} /(\pi Q L)\right)^{M}, \Lambda_{2}=$ $\left(\delta_{\omega} /(\pi Q L)\right)^{M^{\prime}-M}$

For the nonlinear ( $\gamma$ is arbitrary) nondispersive $(\beta=0)$ channel in the leading order in $1 /$ SNR the factorization property was derived in [12]. In the time domain the quantities $P[Y \mid X]$ and $\Lambda$ are factorized:

$$
\begin{aligned}
& P[Y \mid X]=\prod_{j=0}^{M^{\prime}-1} \frac{\delta_{t}}{\pi Q L} \frac{1}{\sqrt{1+\mu_{j}^{2} / 3}} \times \\
& \exp \left\{-\frac{\delta_{t}}{Q L} \frac{\left(1+4 \mu_{j}^{2} / 3\right) x_{j}^{2}-2 \mu_{j} x_{j} y_{j}+y_{j}^{2}}{\left(1+\mu_{j}^{2} / 3\right)}\right\}
\end{aligned}
$$

where $\mu_{j}=\gamma L\left|X\left(t_{j}\right)\right|^{2}$, and $x_{j}+i y_{j}=$ $Y\left(t_{j}\right) e^{-i \phi^{\left(X\left(t_{j}\right)\right)}-i \mu_{j}}-\left|X\left(t_{j}\right)\right|$, with $\phi^{\left(X\left(t_{j}\right)\right)}$ being the phase of the input signal $X\left(t_{j}\right)$. One can see that in the case the conditional probability is the product of the conditional probabilities of $M^{\prime}$ intdependent channels in the time domain, therefore the factorization is obvious.

\section{Appendix C: PERTURBATIVE CALCULATION OF MUTUAL INFORMATION}

In what follows we will calculate the normalization factor $\Lambda_{1}$ in the nonlinear dispersive case in the perturbative expansion in nonlinearity dimensionless parameter $\widetilde{\gamma}=P_{\text {ave }} \gamma L$. We start from the general expression (43) for the mutual information in the leading order in 1/SNR obtained in the main text of the manuscript, see Section III:

$$
I_{P[X]}=H[X]-M+\int d \vec{X}_{1} P_{X}^{(M)}\left[\vec{X}_{1}\right] \log \Lambda_{1}\left[\vec{X}_{1}\right]
$$

where $\vec{X}_{1}$ is $2 M$ dimensional vector corresponding $M$ complex meaning channels in the frequency domain. It is worth noting that this representation is valid for arbitrary nonlinearity but in the leading order in $1 / \mathrm{SNR}$. 
Now we calculate $\Lambda_{1}\left[\vec{X}_{1}\right]$ within the perturbation theory in dimensionless parameter $\tilde{\gamma} \ll 1$ and perform the averaging over Gaussian input signal $\operatorname{PDF} P_{X}^{(M)}$

$$
P_{X}^{(M)}\left[\vec{X}_{1}\right]=P_{G}\left[\vec{X}_{1}\right]=\Lambda_{P} e^{-\left|\vec{X}_{1}\right|^{2} \delta_{\omega} / P},
$$

where the factor $\Lambda_{P}=\left(\delta_{\omega} /(\pi P)\right)^{M}$, is consistent with the normalization condition $\int d \vec{X}_{1} P_{X}^{(M)}\left[\vec{X}_{1}\right]=1$. Here $\delta_{\omega}=W /(2 \pi M)=W^{\prime} /\left(2 \pi M^{\prime}\right)$ is the grid spacing in the frequency domain, see Eq. (A3).

For short we introduce the notation of averaging over $\vec{X}_{1}$ as:

$$
\left\langle f\left[\vec{X}_{1}\right]\right\rangle_{X}=\int d \vec{X}_{1} P_{X}^{(M)}\left[\vec{X}_{1}\right] f\left[\vec{X}_{1}\right]
$$

where $f\left[\vec{X}_{1}\right]$ is an arbitrary function of $\vec{X}_{1}$. Using the notation (C3) the last term in $(\mathrm{C} 1)$ can be written as:

$$
\int d \vec{X}_{1} P_{X}^{(M)}\left[\vec{X}_{1}\right] \log \Lambda_{1}\left[\vec{X}_{1}\right]=\left\langle\log \Lambda_{1}\right\rangle_{X}
$$

Since the PDF (C2) has the Gaussian form we have the following correlator

$$
\left\langle X\left(\omega_{k}\right) \bar{X}\left(\omega_{k^{\prime}}\right)\right\rangle_{X}=P \frac{\delta_{k, k^{\prime}}}{\delta_{\omega}}, \omega_{k}=-\frac{W}{2}+2 \pi \delta_{\omega} k,(
$$

where $k, k^{\prime}=0,1, \ldots, M-1$

From the representation (B19) we obtain the following expression for $\Lambda_{1}\left[\vec{X}_{1}\right]$ in the frequency domain

$$
\begin{aligned}
& \Lambda_{1}\left[\vec{X}_{1}\right]=\int_{\phi(0, \omega)=0}^{\phi(L, \omega)=0}[\mathcal{D} \phi(z, \omega)]_{M} \times \\
& \exp \left\{-\frac{\delta_{\omega}}{Q} \sum_{k=0}^{M-1} \Delta \sum_{n=1}^{N-1} \mathcal{L}_{e f f}\left[\phi\left(z_{n}, \omega_{k}\right)\right]\right\},
\end{aligned}
$$

where the measure reads:

$$
\begin{aligned}
& {[\mathcal{D} \phi(z, \omega)]_{M}=\lim _{\delta_{\omega} \rightarrow 0} \lim _{\Delta \rightarrow 0}\left(\frac{\delta_{\omega}}{\Delta \pi Q}\right)^{M} \times} \\
& \prod_{n=1}^{N-1} \prod_{k=0}^{M-1}\left\{\frac{\delta_{\omega}}{\Delta \pi Q} d \operatorname{Re} \phi\left(z_{n}, \omega_{k}\right) d \operatorname{Im} \phi\left(z_{n}, \omega_{k}\right)\right\} .
\end{aligned}
$$

Now we present the Lagrangian $\mathcal{L}_{\text {eff }}$ as a sum:

$$
\mathcal{L}_{e f f}=\mathcal{L}_{e f f}^{(0)}+\mathcal{L}_{e f f}^{(1)}+\mathcal{L}_{\text {eff }}^{(2)}
$$

Here the first term is the leading order term in $\widetilde{\gamma}$ and it reads

$$
\mathcal{L}_{\text {eff }}^{(0)}\left[\phi\left(z_{n}, \omega_{k}\right)\right]=\left|\left(\partial_{z}-i \beta \bar{\Omega}_{k}^{2}\right) \phi\left(z_{n}, \omega_{k}\right)\right|^{2},
$$

where we have introduced the notation

$$
\bar{\Omega}_{k}=2 \sin \left[\frac{\pi k}{M}\right] \frac{M}{T}=2 M \delta_{\omega} \sin \left[\frac{\pi k}{M}\right]=\frac{W}{\pi} \sin \left[\frac{\pi k}{M}\right],
$$

see Eq. (A14). In the continuous limit $M \rightarrow \infty$ we will assume that $\bar{\Omega}_{k} \approx 2 \pi \delta_{\omega} k=\omega_{k}+W / 2$. The second term in the Lagrangian (C8) reads

$$
\begin{aligned}
& \mathcal{L}_{\text {eff }}^{(1)}\left[\phi\left(z_{n}, \omega_{k}\right)\right]=2 \gamma \operatorname{Im}\left\{\left[\left(\partial_{z}+i \beta \bar{\Omega}_{k}^{2}\right) \bar{\phi}\left(z_{n}, \omega_{k}\right)\right]\right. \\
& \delta_{\omega}^{2} \sum_{k_{1}=0}^{M-1} \sum_{k_{2}=0}^{M-1} \sum_{k_{3}=0}^{M-1} \Delta_{(M)}\left(k_{1}+k_{2}-k_{3}-k\right) \times \\
& {\left[2 \phi\left(z_{n}, \omega_{k_{1}}\right) \Phi\left(z_{n}, \omega_{k_{2}}\right) \bar{\Phi}\left(z_{n}, \omega_{k_{3}}\right)+\right.} \\
& \left.\left.\bar{\phi}\left(z_{n}, \omega_{k_{3}}\right) \Phi\left(z_{n}, \omega_{k_{1}}\right) \Phi\left(z_{n}, \omega_{k_{2}}\right)\right]\right\}
\end{aligned}
$$

where $\Delta_{(M)}\left(k_{1}+k_{2}-k_{3}-k\right)$ is defined in Eq. (A15). In the following calculation we will use the function $\Phi\left(z_{n}, \omega_{k}\right)$ in the leading and next-to-leading order in $\gamma$ : $\Phi\left(z, \omega_{k}\right) \approx \Phi^{(0)}\left(z, \omega_{k}\right)+\Phi^{(1)}\left(z, \omega_{k}\right)$, where

$$
\begin{aligned}
& \Phi^{(0)}\left(z, \omega_{k^{\prime}}\right)=e^{i \beta \bar{\Omega}_{k^{\prime}}^{2} z} X\left(\omega_{k^{\prime}}\right), \\
& \Phi^{(1)}\left(z, \omega_{k^{\prime}}\right)=i \gamma e^{i \beta \bar{\Omega}_{k^{\prime}}^{2} z} \delta_{\omega}^{2} \sum_{k_{1}^{\prime}=0}^{M-1} \sum_{k_{2}^{\prime}=0}^{M-1} \sum_{k_{3}^{\prime}=0}^{M-1} K(\mu, z) \times \\
& X\left(\omega_{k_{1}^{\prime}}\right) X\left(\omega_{k_{2}^{\prime}}\right) \bar{X}\left(\omega_{k_{3}^{\prime}}\right) \Delta_{(M)}\left(k_{1}^{\prime}+k_{2}^{\prime}-k_{3}^{\prime}-k^{\prime}\right),
\end{aligned}
$$

for $k^{\prime}=0,1, \ldots, M-1$, here $\bar{\Omega}_{k^{\prime}}$ is defined in Eq. (C10), $\mu=\mu\left(k^{\prime}, k_{1}^{\prime}, k_{2}^{\prime}, k_{3}^{\prime}\right)=i \beta L\left(\bar{\Omega}_{k^{\prime}}^{2}+\bar{\Omega}_{k_{3}^{\prime}}^{2}-\bar{\Omega}_{k_{1}^{\prime}}^{2}-\bar{\Omega}_{k_{2}^{\prime}}^{2}\right)$, and $K(\mu, z)=\left(1-e^{-\mu z / L}\right) / \mu$.

The third term in the Lagrangian reads

$$
\begin{aligned}
& \mathcal{L}_{\text {eff }}^{(2)}\left[\phi\left(z_{n}, \omega_{k}\right)\right]=\gamma^{2} \delta_{\omega}^{2} \sum_{k_{1}=0}^{M-1} \sum_{k_{2}=0}^{M-1} \sum_{k_{3}=0}^{M-1} \Delta_{(M)}\left(k_{1}+k_{2}-k_{3}-k\right) \delta_{\omega}^{2} \sum_{k_{1}^{\prime}=0}^{M-1} \sum_{k_{2}^{\prime}=0}^{M-1} \sum_{k_{3}^{\prime}=0}^{M-1} \Delta_{(M)}\left(k_{1}^{\prime}+k_{2}^{\prime}-k_{3}^{\prime}-k\right) \times \\
& \left(2 \phi\left(z_{n}, \omega_{k_{1}}\right) \Phi\left(z_{n}, \omega_{k_{2}}\right) \bar{\Phi}\left(z_{n}, \omega_{k_{3}}\right)+\bar{\phi}\left(z_{n}, \omega_{k_{3}}\right) \Phi\left(z_{n}, \omega_{k_{1}}\right) \Phi\left(z_{n}, \omega_{k_{2}}\right)\right) \times \\
& {\left[2 \bar{\phi}\left(z_{n}, \omega_{k_{1}^{\prime}}\right) \bar{\Phi}\left(z_{n}, \omega_{k_{2}^{\prime}}\right) \Phi\left(z_{n}, \omega_{k_{3}^{\prime}}\right)+\phi\left(z_{n}, \omega_{k_{3}^{\prime}}\right) \bar{\Phi}\left(z_{n}, \omega_{k_{1}^{\prime}}\right) \bar{\Phi}\left(z_{n}, \omega_{k_{2}^{\prime}}\right)\right]}
\end{aligned}
$$

Let us stress that everywhere the derivatives with 
other fields $\phi\left(z_{n}, \omega_{k}\right)$ in the same point $z_{n}=$ $n \Delta$, are assumed as the difference derivatives in the "causative" manner: $f\left[\partial_{z} \phi\left(z_{n}, \omega_{k}\right) ; \phi\left(z_{n}, \omega_{k}\right)\right]=$ $f\left[\left(\phi\left(z_{n+1}, \omega_{k}\right)-\phi\left(z_{n}, \omega_{k}\right)\right) / \Delta ; \phi\left(z_{n}, \omega_{k}\right)\right]$, as provided by our approach [19]. In what follows for brevity sake we will write a sum over $z\left(\Delta \sum_{n=1}^{N-1} \ldots\right)$ as an integral $\left(\int_{0}^{L} d z \ldots\right)$.

We present the perturbative expansion in $\widetilde{\gamma}$ of the normalization factor $\Lambda_{1}$, see Eq. (C6), in the form:

$$
\Lambda_{1}=\Lambda_{1}^{(0)}+\Lambda_{1}^{(1)}+\Lambda_{1}^{(2)}+\mathcal{O}\left(\widetilde{\gamma}^{3}\right),
$$

where $\Lambda_{1}^{(m)}$ is of order of $\widetilde{\gamma}^{m}$.

Thus the last term in the expression (C1) for the mutual information has the following expansion in $\widetilde{\gamma}$ :

$$
\begin{aligned}
& \left\langle\log \Lambda_{1}\right\rangle_{X}=\left\langle\log \Lambda_{1}^{(0)}\right\rangle_{X}+\left\langle\frac{\Lambda_{1}^{(1)}}{\Lambda_{1}^{(0)}}\right\rangle_{X}+ \\
& \left(\left\langle\frac{\Lambda_{1}^{(2)}}{\Lambda_{1}^{(0)}}\right\rangle_{X}-\frac{1}{2}\left\langle\left(\frac{\Lambda_{1}^{(1)}}{\Lambda_{1}^{(0)}}\right)^{2}\right\rangle_{X}\right)+\mathcal{O}\left(\widetilde{\gamma}^{3}\right) .
\end{aligned}
$$

The order $\gamma^{0}$. Retaining only the first term (C9) in the exponent in Eq. (C6) we arrive at:

$$
\begin{aligned}
& \Lambda_{1}^{(0)}=\int_{\phi(0, \omega)=0}^{\phi(L, \omega)=0}[\mathcal{D} \phi(z, \omega)]_{M} \times \\
& e^{-\frac{\delta_{\omega}}{Q} \sum_{k=0}^{M-1} \int_{0}^{L} d z\left|\left(\partial_{z}-i \beta \bar{\Omega}_{k}^{2}\right) \phi\left(z, \omega_{k}\right)\right|^{2}}=\left(\frac{\delta_{\omega}}{\pi Q L}\right)^{M} .
\end{aligned}
$$

By taking into account that for the Gaussian distribution (C2) the input entropy has the form

$$
H[X]=M-M \log \left[\delta_{\omega} /(\pi P)\right],
$$

the expression for $\log \Lambda_{1}^{(0)}$ results in the leading (Shannon's) contribution $M \log P /(Q L)$ to the mutual information.

For the path-integral (C6) we introduce the averaging $\langle\ldots\rangle_{\phi}$ over fields $\phi(z, \omega)$ defined as

$$
\begin{aligned}
& \langle(\ldots)\rangle_{\phi}=\frac{1}{\Lambda_{1}^{(0)}} \int_{\phi(0, \omega)=0}^{\phi(L, \omega)=0}[\mathcal{D} \phi(z, \omega)]_{M}(\ldots) \times \\
& \exp \left\{-\frac{\delta_{\omega}}{Q} \sum_{k=0}^{M-1} \int_{0}^{L} d z\left|\left(\partial_{z}-i \beta \bar{\Omega}_{k}^{2}\right) \phi\left(z, \omega_{k}\right)\right|^{2}\right\} .
\end{aligned}
$$

The paired correlator for this averaging can be calculated explicitly:

$$
\begin{aligned}
& \left\langle\phi\left(z, \omega_{k}\right) \bar{\phi}\left(z^{\prime}, \omega_{k^{\prime}}\right)\right\rangle_{\phi}= \\
& -\frac{Q}{\delta_{\omega}} \delta_{k, k^{\prime}} G\left(z, z^{\prime}\right) \exp \left[i \beta \bar{\Omega}_{k}^{2}\left(z-z^{\prime}\right)\right], \\
& \left\langle\phi\left(z, \omega_{k}\right) \phi\left(z^{\prime}, \omega_{k^{\prime}}\right)\right\rangle_{\phi}=0
\end{aligned}
$$

where

$$
G\left(z, z^{\prime}\right)=z \frac{z^{\prime}-L}{L} \theta\left(z^{\prime}-z\right)+z^{\prime} \frac{z-L}{L} \theta\left(z-z^{\prime}\right)
$$

is the Green function of the operator $\partial_{z}^{2}$ with the boundary conditions $G\left(0, z^{\prime}\right)=G\left(L, z^{\prime}\right)=0$. In the notations (C19) we can present the mutual information (C1) as a sum of Shannon's contribution and the term which is accountable for the impact of nonlinearity:

$$
\begin{gathered}
I_{P[X]}=M \log [P /(Q L)]+ \\
\left\langle\log \left\langle\exp \left\{-\frac{S_{n l}[\phi(z, \omega)]}{Q}\right\}\right\rangle_{\phi}\right\rangle_{X}, \\
S_{n l}[\phi(z, \omega)]=\int_{0}^{L} d z \delta_{\omega} \sum_{k=0}^{M-1}\left(\mathcal{L}_{\text {eff }}^{(1)}+\mathcal{L}_{\text {eff }}^{(2)}\right) .
\end{gathered}
$$

The order $\gamma^{1}$. For the second term in the r.h.s. of Eq. (C16) we have

$$
\begin{aligned}
& \left\langle\frac{\Lambda_{1}^{(1)}}{\Lambda_{1}^{(0)}}\right\rangle_{X}= \\
& -\left\langle\frac{1}{Q} \int_{0}^{L} d z \delta_{\omega} \sum_{k=0}^{M-1}\left\langle\left.\mathcal{L}_{\text {eff }}^{(1)}\left[\phi\left(z, \omega_{k}\right)\right]\right|_{\Phi=\Phi^{(0)}}\right\rangle_{\phi}\right\rangle_{X},
\end{aligned}
$$

where $\mathcal{L}_{\text {eff }}^{(1)}$ is given by the expression (C11) with the function $\Phi\left(z, \omega_{k}\right)$ being replaced with the zero order term $\Phi^{(0)}\left(z, \omega_{k}\right)$, see Eq. (C12). Performing the averaging $\langle\ldots\rangle_{\phi}$ by virtue of Eq. (C20) we obtain that the contribution (C23) vanishes as the imaginary part of the real value:

$$
\left\langle\frac{\Lambda_{1}^{(1)}}{\Lambda_{1}^{(0)}}\right\rangle_{X}=0
$$

It means that there are no corrections to the mutual information (C1) of order of $\widetilde{\gamma}$.

The order $\gamma^{2}$. Let us consider the first term in Eq. (C16) of order of $\gamma^{2}$. There are three contributions to the quantity $\Lambda_{1}^{(2)}$ :

$$
\Lambda_{1}^{(2)}=\Lambda_{1}^{(2.1)}+\Lambda_{1}^{(2.2)}+\Lambda_{1}^{(2.3)} .
$$

The first contribution $\Lambda_{1}^{(2.1)}$ in (C25) comes from the next-to-leading order expansion of the function $\Phi(z, \omega)$ in the expression (C11) for $\mathcal{L}_{\text {eff }}^{(1)}$ : see Eq. (C13). For this contribution we can write the following representation 


$$
\begin{gathered}
\frac{\Lambda_{1}^{(2.1)}}{\Lambda_{1}^{(0)}}=-\frac{4 \gamma}{Q} \operatorname{Im}\left\langle\int_{0}^{L} d z \delta_{\omega} \sum_{k=0}^{M-1}\left(\partial_{z}+i \beta \bar{\Omega}_{k}^{2}\right) \bar{\phi}\left(z, \omega_{k}\right) \delta_{\omega}^{2} \sum_{k_{1}=0}^{M-1} \sum_{k_{2}=0}^{M-1} \sum_{k_{3}=0}^{M-1} \Delta_{(M)}\left(k_{1}+k_{2}-k_{3}-k\right) \times\right. \\
\left.\left[\phi\left(z, \omega_{k_{1}}\right)\left(\Phi^{(0)}\left(z, \omega_{k_{2}}\right) \bar{\Phi}^{(1)}\left(z, \omega_{k_{3}}\right)+\Phi^{(1)}\left(z, \omega_{k_{2}}\right) \bar{\Phi}^{(0)}\left(z, \omega_{k_{3}}\right)\right)+\bar{\phi}\left(z, \omega_{k_{3}}\right) \Phi^{(0)}\left(z, \omega_{k_{1}}\right) \Phi^{(1)}\left(z, \omega_{k_{2}}\right)\right]\right\rangle_{\phi}
\end{gathered}
$$

where $\Phi^{(0)}$ is given by Eq. (C12), and $\Phi^{(1)}$ is given by Eq. (C13). After averaging $\langle\ldots\rangle_{\phi}$ with the help of Eq. (C20) we use Eqs. (C12) and (C13) to find $\left\langle\Lambda_{1}^{(2.1)} / \Lambda_{1}^{(0)}\right\rangle_{X}$. From the Wick theorem [25, 26] with the correlator (C5) applied to the averaging over $X$ we find that all pairings cancel with each other:

$$
\left\langle\frac{\Lambda_{1}^{(2.1)}}{\Lambda_{1}^{(0)}}\right\rangle_{X}=0
$$

The second contribution $\Lambda_{1}^{(2.2)}$ in $(\mathrm{C} 25)$ comes from $\mathcal{L}_{\text {eff }}^{(2)}$, see Eq. (C14), where $\Phi(z, \omega)$ is considered in the leading order in $\gamma$, see Eq. (C12). For this contribution we can write the following representation

$$
\frac{\Lambda_{1}^{(2.2)}}{\Lambda_{1}^{(0)}}=-\frac{1}{Q} \int_{0}^{L} d z \delta_{\omega} \sum_{k=0}^{M-1}\left\langle\left.\mathcal{L}_{\text {eff }}^{(2)}\left[\phi\left(z, \omega_{k}\right)\right]\right|_{\Phi=\Phi^{(0)}}\right\rangle_{\phi} .
$$

The averaging $\langle\ldots\rangle_{\phi}$ is straightforward. To find the quantity $\left\langle\Lambda_{1}^{(2.2)} / \Lambda_{1}^{(0)}\right\rangle_{X}$ we use the Wick theorem $[25$, 26 ] with the correlator Eq. (C5). After Wick pairing we verify that the dependence on $\beta$ vanishes for this quantity. Finally, we have

$$
\left\langle\frac{\Lambda_{1}^{(2.2)}}{\Lambda_{1}^{(0)}}\right\rangle_{X}=-\frac{5}{3} M \widetilde{\gamma}^{2}
$$

Here we have used the value of the integral $\int_{0}^{L} G(z, z) d z=-L^{2} / 6$ with the Green function (C21). When obtaining (C29) we also used that $\sum_{k=0}^{M-1} \Delta_{(M)}\left(k_{1}+k_{2}-k_{3}-k\right)=1$, where $0 \leq k_{i} \leq M-1$, see Eq. (A15).

The third contribution $\Lambda_{1}^{(2.3)}$ in (C25) comes from the expansion of the exponent in Eq. (C22) of order of $\left[\mathcal{L}_{\text {eff }}^{(1)}\right]^{2}:$

$$
\begin{aligned}
& \frac{\Lambda_{1}^{(2.3)}}{\Lambda_{1}^{(0)}}=\frac{1}{2 Q^{2}} \int_{0}^{L} d z_{1} \int_{0}^{L} d z_{2} \delta_{\omega}^{2} \sum_{k=0}^{M-1} \sum_{k^{\prime}=0}^{M-1} \\
& \left\langle\left.\mathcal{L}_{\text {eff }}^{(1)}\left[\phi\left(z_{1}, \omega_{k}\right)\right] \mathcal{L}_{\text {eff }}^{(1)}\left[\phi\left(z_{2}, \omega_{k}^{\prime}\right)\right]\right|_{\Phi=\Phi^{(0)}}\right\rangle_{\phi},
\end{aligned}
$$

where $\mathcal{L}_{\text {eff }}^{(1)}$ appears in Eq. (C30) through the representation (C11) with $\Phi(z, \omega)$ being replaced with the lead- ing order term $\Phi^{(0)}\left(z, \omega_{k}\right)$, see Eq. (C12). The calculation of the contribution $\left\langle\Lambda_{1}^{(2.3)} / \Lambda_{1}^{(0)}\right\rangle_{X}$ to the r.h.s. of Eq. (C16) is the most cumbersome but straightforward. Here we present the result of the calculation:

$$
\left\langle\frac{\Lambda_{1}^{(2.3)}}{\Lambda_{1}^{(0)}}\right\rangle_{X}=M \widetilde{\gamma}^{2}\left[\frac{5}{3}-\frac{1}{3} g(\widetilde{\beta})\right],
$$

where the function $g(\widetilde{\beta})\left(\widetilde{\beta}=\beta L W^{2}\right.$ is dimensionless dispersion parameter) can be presented in our discretization scheme in the form of a triple sum:

$$
\begin{aligned}
& g(\widetilde{\beta})= \\
& \frac{1}{M^{3}} \sum_{k_{1}, k_{2}, k_{3}=0}^{M-1} F\left(\frac{\widetilde{\beta}}{2}\left[\bar{\Omega}_{k_{1}}^{2}+\bar{\Omega}_{k_{2}}^{2}-\bar{\Omega}_{k_{3}}^{2}-\bar{\Omega}_{k_{1}+k_{2}-k_{3}}^{2}\right]\right) .
\end{aligned}
$$

The function $F(\mu)$ in Eq. (C32) is the result of integration of the derivatives of the dimensionless Green function, $G_{0}\left(\zeta_{1}, \zeta_{2}\right)=\zeta_{1}\left(\zeta_{2}-1\right) \theta\left(\zeta_{2}-\zeta_{1}\right)+\zeta_{2}\left(\zeta_{1}-1\right) \theta\left(\zeta_{1}-\zeta_{2}\right)$, see Eq. (C21),

$$
\begin{aligned}
& F(\mu)=-12 \int_{0}^{1} d \zeta_{1} \int_{0}^{1} d \zeta_{2} \frac{\partial G_{0}\left(\zeta_{1}, \zeta_{2}\right)}{\partial \zeta_{1}} \frac{\partial G_{0}\left(\zeta_{1}, \zeta_{2}\right)}{\partial \zeta_{2}} \times \\
& e^{-2 i \mu\left(\zeta_{1}-\zeta_{2}\right)}=3 \frac{\mu^{2}-\sin ^{2}(\mu)}{\mu^{4}}= \\
& 4 ! \sum_{s=0}^{\infty} \frac{(-1)^{s}(2 \mu)^{2 s}}{(2 s+4) !}
\end{aligned}
$$

and for convenience it is normalized as $F(0)=1$.

The last term of order of $\gamma^{2}$ in Eq. (C16) is zero for the same reasons as in Eq. (C24).

$$
\left\langle\left(\frac{\Lambda_{1}^{(1)}}{\Lambda_{1}^{(0)}}\right)^{2}\right\rangle_{X}=0
$$

Now we call together all terms in Eq. (C16): Eqs. (C17), (C24), (C27), (C29), (C31), and (C34). Finally, we obtain the following expression for the mutual information in the leading order in $1 / \mathrm{SNR}$ for the Gaussian PDF (C2):

$$
I_{P_{G}[X]}=M\left\{\log \mathrm{SNR}-\frac{\widetilde{\gamma}^{2}}{3} g(\widetilde{\beta})\right\}+\mathcal{O}\left(\widetilde{\gamma}^{4}\right),
$$


where $M$ is the number of complex meaning channels. This number is implied to be large $M \gg 1$. In the continuous limit of sufficiently large $M$ we present $g(\widetilde{\beta})$ through the integral:

$$
\begin{aligned}
& g(\widetilde{\beta})= \\
& \int_{0}^{1} d x_{1} \int_{0}^{1} d x_{2} \int_{0}^{1} d x_{3} F\left(\frac{\widetilde{\beta}}{4}\left(x_{1}-x_{3}\right)\left(x_{2}-x_{3}\right)\right),
\end{aligned}
$$

where we have used the expansion of $\bar{\Omega}_{k}$, see Eq. (C10), when the argument of the sinus is close to zero or to $\pi$, and we have used the identity $x_{1}^{2}+x_{2}^{2}-x_{3}^{2}-\left(x_{1}+x_{2}-\right.$ $\left.x_{3}\right)^{2}=-2\left(x_{1}-x_{3}\right)\left(x_{2}-x_{3}\right)$. To calculate analytically the integral (C36) one can perform the series expansion (C33) for $F(\mu)$ and the term by term integration for the polynomials $\left(x_{1}-x_{3}\right)^{2 n}\left(x_{2}-x_{3}\right)^{2 n}$ :

$$
\begin{aligned}
& g(\widetilde{\beta})=4 ! \sum_{n=0}^{\infty} a_{n} \frac{(-1)^{n}(\widetilde{\beta} / 2)^{2 n}}{(2 n+4) !}, \\
& a_{n}=\int_{0}^{1} d x_{1} \int_{0}^{1} d x_{2} \int_{0}^{1} d x_{3}\left(x_{1}-x_{3}\right)^{2 n}\left(x_{2}-x_{3}\right)^{2 n}= \\
& \frac{2}{(1+2 n)^{2}}\left(\frac{1}{3+4 n}+\frac{\Gamma(2+2 n)^{2}}{\Gamma(4+4 n)}\right) .
\end{aligned}
$$

The series (C37) can be calculated in the term of the generalized hypergeometric functions.

For $\widetilde{\beta}=0$ we have $g(0)=1$ and we arrive at the result [12] for the nondispersive channel in expansion in $\widetilde{\gamma}$ :

$$
I_{P[X]}=M \log \mathrm{SNR}-M \frac{\widetilde{\gamma}^{2}}{3}+\mathcal{O}\left(\widetilde{\gamma}^{4}\right)
$$

For large $\widetilde{\beta}$ we can consider the asymptotics of the function $g(\widetilde{\beta})$ obtained from the integral representation (C36):

$$
g(\widetilde{\beta})=\frac{16 \pi}{\widetilde{\beta}}\left(\log \frac{\widetilde{\beta}}{2}+\gamma_{E}-\frac{23}{6}\right)+\mathcal{O}\left(\widetilde{\beta}^{-3 / 2}\right)
$$

[1] K. Turitsyn, V. Chernyak, M. Chertkov, and A. Puliafito, Phys. Rev. Lett. 98, 180603 (2007).

[2] C. Shannon, A mathematical theory of communication, Bell System Techn. J., 27, 3, 379 (1948); 27, 4, 623 (1948).

[3] P. P. Mitra and J. B. Stark, Nature 411, 1027 (2001).

[4] R.-J. Essiambre, G. J. Foschini, G. Kramer, and P. J. Winzer, Phys. Rev. Lett. 101, 163901 (2008).

[5] R.-J. Essiambre, G. Kramer, P. J. Winzer, G. J. Foschini, and B. Goebel, J. of Lightwave Technol. 28, 0733 (2010).

[6] R. Killey and C. Behrens, J. Mod. Opt. 58, 1, (2011).

[7] A. D. Ellis and J. Zhao, Impact of Nonlinearities on Fiber Optic Communications, Springer, New York, (2011).

[8] E. Agrell, arXiv: 1108.0391v3.

[9] E. Agrell, Nonlinear Fiber Capacity, presented at the Eur. Conf. Opt. Commun. London U.K., paper We.4.D.3 (2013).

[10] E. Agrell, A. Alvarado, G. Durisi, M. Karlsson, arXiv:1403.3339

[11] K. S. Turitsyn, S. A. Derevyanko, I. V. Yurkevich, and S. K. Turitsyn, Phys. Rev. Lett. 91, 203901 (2003).

[12] I. S. Terekhov, A. V. Reznichenko, Ya. A. Kharkov, and S. K. Turitsyn, arXiv: 1508.05774.

[13] M. I. Yousefi, F. R. Kschischang, IEEE transactions on information theory, V. 57, No. 11, 7522 (2011).

[14] H. A. Haus, J. Opt. Soc. Am. B, V. 8, No 5, 1122 (1991).

[15] A. Mecozzi, J.Lightw. Technol., V. 12, No. 11, 1993 (1994).

[16] E. Iannoe, F. Matera, A. Mecozzi, and M. Settembre, Nonlinear Optical Communication Networks, John Wiley \& Sons, New York, (1998).
[17] S. K. Turitsyn, S. B. Medvedev, M. P. Fedoruk, and E. G. Turitsyna, Phys. Rev. E 61, 3127 (2000).

[18] C. E. Shannon, Communication in the presence of noise, Proc. Institute of Radio Engineers, vol. 37, 1, (1949). V. A. Kotelnikov, On the capacity of the ether and wire in Telecommunications, Proceedings of the First All-Union Congress on Technical Reconstruction of Communication. Union Energy Committee. (1933), In Russian.

[19] I. S. Terekhov, S. S. Vergeles, and S. K. Turitsyn, Phys. Rev. Lett. 113, 230602 (2014).

[20] M. Kac, G. E. Uhlenbeck, A. R. Hibbs, Probability and related topics in physical sciences, Interscience Publishers, New York, (1957).

[21] R. P. Feynman, A. R. Hibbs, Quantum mechanics and path integrals, McGraw-Hill Book Company, New York, (1965).

[22] P. C. Martin, E. D. Siggia, and H. A. Rose, Phys. Rev. A, 8, 423 (1973).

[23] L. D. Landau and E. M. Lifshitz, Quantum mechanichs: non-relativistic theory, Pergamon Press Inc., New York 10523 U.S.A., 1981.

[24] S. Novikov, S. V. Manakov, L. P. Pitaevskii, V. E. Zakharov, Theory of Solitons: The Inverse Scattering Method (Monographs in Contemporary Mathematics), Springer, Rumford, ME, U.S.A. (1984).

[25] M. E. Peskin, D. V. Schroeder, An Introduction to Quantum Field Theory, Westview Press, 1995. Chapter 12.

[26] C. Itzykson, J.B. Zuber, Quantum Field Theory, McGraw-Hill (1980). 